\title{
Preexisting subtype immunodominance shapes memory B cell recall response to influenza vaccination
}

\author{
Rodrigo B. Abreu, ${ }^{1}$ Greg A. Kirchenbaum, ${ }^{1}$ Emily F. Clutter, ${ }^{1}$ Giuseppe A. Sautto, ${ }^{1}$ and Ted M. Ross ${ }^{1,2}$ \\ ${ }^{1}$ Center for Vaccines and Immunology and ${ }^{2}$ Department of Infectious Diseases, University of Georgia, Athens, Georgia, USA.
}

\begin{abstract}
Influenza is a highly contagious viral pathogen with more than 200,000 cases reported in the United States during the 2017-2018 season. Annual vaccination is recommended by the World Health Organization, with the goal to reduce influenza severity and transmission. Currently available vaccines are about $\mathbf{6 0} \%$ effective, and vaccine effectiveness varies from season to season, as well as between different influenza subtypes within a single season. Immunological imprinting from early-life influenza infection can prominently shape the immune response to subsequent infections. Here, the impact of preexisting B cell memory in the response to quadrivalent influenza vaccine was assessed using blood samples collected from healthy subjects ( $18-85$ years old) prior to and 21-28 days following influenza vaccination. Influenza vaccination increased both HA-specific antibodies and memory B cell frequency. Despite no apparent differences in antigenicity between vaccine components, most individuals were biased toward one of the vaccine strains. Specifically, responses to H3N2 were reduced in magnitude relative to the other vaccine components. Overall, this study unveils a potentially new mechanism underlying differential vaccine effectiveness against distinct influenza subtypes.
\end{abstract}

Conflict of interest: TMR has research funding from Sanofi Pasteur Inc. TMR is also supported, in part, by the Georgia Research Alliance as an Eminent Scholar.

Copyright: ( 2020 , American Society for Clinical Investigation.

Submitted: July 29, 2019 Accepted: November 20, 2019 Published: January 16, 2020.

Reference information: /CI Insight. 2020;5(1):e132155.

https://doi.org/10.1172/jci. insight.132155.

\section{Introduction}

Influenza remains one of the most contagious infectious diseases. Approximately, 25-50 million people suffer from influenza-like illness in the United States annually, leading to almost 1 million hospitalizations (1). Globally, the World Health Organization (WHO) estimates 250,000-500,000 mortalities associated with secondary respiratory complications due to influenza virus infection every year. The elderly (defined as people over 65 years of age) have higher rates of mortality related to influenza infection than adults or children (2-5). Influenza vaccination elicits antibody responses in the elderly that are often compromised and wane before the next season, leading to poor protection in this highrisk population (6). Annual influenza vaccination is recommended for these high risk groups but is most effective in children and young adults $(7,8)$.

Despite extensive efforts to develop a universal influenza vaccine, influenza viruses undergo change (drift) from season to season, and the current licensed influenza vaccines offer variable effectiveness due to seasonal antigenic variants (9). There are 5 highly variable antigenic sites in the hemaglutinin-globular (HA-globular) head of influenza A viruses (IAVs) (i.e., H1N1 and H3N2), as well as influenza B viruses (IBVs) (10-12). These regions of the HA protein are often targeted by the humoral immune system to neutralize viral entry into cells. Antibodies endowed with receptor-blocking activity can efficiently neutralize virus in vivo but frequently lead to strain-specific protection with narrow reactivity against other strains. Thus, vaccine-induced antibody breadth against historical, previously circulating, or antigenically related strains has the potential to increase the effectiveness of trivalent influenza vaccines (TIVs) and quadrivalent influenza vaccines (QIVs) but is highly dependent on preimmune status (13). Recently, broadly reactive antibodies directed to the conserved stem region of the HA protein have been shown to protect and ameliorate disease during influenza infection, independent of receptor-blocking activity (14-16). However, the globular head portion of 
the HA protein is highly immunodominant compared with the HA stem region (17), which has complicated efforts toward development of stem-based vaccines (18-21).

Seasonal influenza A and B viruses cocirculate worldwide in the human population with unpredictable patterns. Consequently, the first influenza monovalent vaccines developed in 1930 were quickly updated in 1940 to include an influenza B strain and later to a trivalent formulation with a second IAV strain (22). Initial reports showed that polyvalency significantly decreased effectiveness compared with monovalent influenza vaccines against matched influenza virus infections; however, the exact immunological mechanism behind this phenomenon was not understood at the time $(23,24)$. In this century, TIV was again updated to a quadrivalent formulation with inclusion of a second influenza B strain to cover the independently evolving IBV lineages $(12,25,26)$.

Polyvalent vaccination poses several challenges (27). During production stage, polyvalent vaccines often increase the time and costs of manufacturing, and they require additional safety and quality control testing. During the clinical stage, polyvalency can alter antigen immunogenicity due to cross-reactive epitopes and immunogenic hierarchy $(28,29)$. Other multivalent vaccines such as those for dengue, Streptococcus pneumoniae, and human papillomaviruses have revealed these challenges (30). Antigenic immunodominance during influenza infection has also been previously reported (31-33), but heterosubtypic competition between vaccine strains has not been experimentally addressed.

HA and neuraminidase (NA) are the 2 main surface glycoproteins expressed in influenza viral particles, and they are major targets of the antibody response elicited following influenza infection (34). Preexisting immunity prominently modulates these serological responses to subsequent influenza infections (35-40), but the impact of preimmune status on B cell memory responses to influenza vaccination is less understood.

Current influenza vaccines provide limited protection, even in well-matched years (9), with particularly low efficacy in high-risk populations (41). Different production systems (i.e., cell-based vs. embryonated chicken egg) and inactivation/purification methods can introduce genetic and structural changes with severe impacts in antigenicity (31). Moreover, unadjuvanted inactivated vaccines fail to generate strong $\mathrm{T}$ cell-dependent responses and therefore rely on the recall of preexisting immunity, which is extremely diverse in the human population (42). To this end, there is an urgent need to better understand the human immune response to influenza vaccination so we can fully comprehend the underlying shortcomings and pitfalls of current influenza vaccines.

This study investigates the induction of plasmablast and B cell memory responses elicited by the 20162017 Fluzone seasonal influenza vaccine in a cohort of healthy subjects ranging in age and influenza life history (Figure 1). Serum and peripheral blood mononuclear cells (PBMC) samples were analyzed with the goal of (a) determining how vaccination against circulating influenza virus strains is influenced by preexisting serologic immunity and (b) whether age-dependent seroconversion differences accounted for reduced vaccine effectiveness (VE). Here, we present evidence supporting preexisting serological subtype immunodominance against vaccinal HA components, which varied between age groups. Moreover, despite induction of HA-specific antibody titers, seasonal influenza vaccination did not overcome preexisting subtype immunodominance. Finally, despite recall of preexisting B cell memory, the H3N2 vaccine strain elicited a subdominant response.

\section{Results}

Demographics of volunteers. Subjects were recruited from the Athens, Georgia, USA, metropolitan region during the 2016-2017 influenza season. Twice as many women than men were enrolled in the study. Approximately $75 \%$ of the subjects were self-identified as White, with $10 \%$ classified as African American/ Black and $6 \%-8 \%$ self-identified as Hispanic/Latino or Asian. Subjects ranged in age from 18-85 years old (y.o.) with $63 \%$ of the subjects between the age of $18-34$ y.o. (young adults). In the elderly (65-85 y.o.), 67\% of the subjects were male (Table 1).

Inactivated split-virion influenza vaccine induces seroprotective antibodies against the 4 vaccine components. Receptor blocking antibodies (RB-Abs) against influenza viruses are traditionally assessed through inhibition of erythrocyte hemaglutinaition inhibition (HAI) (43). To assess the impact of QIV on the levels of RB-Abs, the HAI activity of serum samples from healthy volunteers prior to and 21-28 days following QIV was tested against the 4 vaccine strains. QIV significantly increased serological HAI activity, measured by endpoint titer, against each of the 4 vaccine components (mean D21-D0 $=176 \pm 24 ; 189 \pm 22$; $277 \pm 48 ; 218 \pm 39$ for $\mathrm{H} 1 \mathrm{~N} 1, \mathrm{H} 3 \mathrm{~N} 2, \mathrm{~B}_{\text {vic }}$, and $\mathrm{B}_{\text {yam }}$, respectively) following vaccination (Figure $\left.2 \mathrm{~A}\right)$. Influ- 
Table 1. Demographics of volunteers

\begin{tabular}{|c|c|c|c|c|c|c|}
\hline \multirow{2}{*}{\multicolumn{2}{|c|}{$\begin{array}{c}\text { Season } \\
\text { UGA }\end{array}$}} & \multirow{2}{*}{$\begin{array}{c}\text { 2016-17 } \\
\text { Total }\end{array}$} & \multicolumn{4}{|c|}{ Age groups } \\
\hline & & & $18-34$ & $35-49$ & $50-64$ & $65-80+$ \\
\hline \multicolumn{2}{|c|}{ Subject (\#) } & 148 & 93 & 19 & 21 & 15 \\
\hline $\operatorname{Sex}(\%)$ & Male & $39 \%$ & $30 \%$ & $37 \%$ & $57 \%$ & $67 \%$ \\
\hline \multirow{4}{*}{ Ethnicity (\%) } & White & $75 \%$ & $45 \%$ & $8 \%$ & $12 \%$ & $9 \%$ \\
\hline & Black/AA & $10 \%$ & $7 \%$ & $2 \%$ & $1 \%$ & $1 \%$ \\
\hline & Al & $1 \%$ & $0 \%$ & $0 \%$ & $0 \%$ & $0 \%$ \\
\hline & Total & & $63 \%$ & $13 \%$ & $13 \%$ & $10 \%$ \\
\hline
\end{tabular}

AA, African American; Al, American Indian.

enza VE is highly age dependent $(13,43,44)$. When stratifying by age, only young adults (18-35 y.o.) had a significant increase in HAI titers against all vaccine strains (mean D21-D0 = of 164 \pm 24 ) (Figure 2B). Vaccine-induced HAI titers in middle-aged adults (35-50 y.o.) were dependent on the vaccine strain (Figure 2, C and D). In contrast, serological HAI titers against the 4 vaccine strains were not significantly increased in elderly adults ( $>65$ y.o.) following vaccination (Figure $2 \mathrm{E}$ ). Seroprotection is generally correlated with an HAI titer $\geq 1: 40$. Following QIV, more than $75 \%$ of the cohort was seropositive against all vaccine strains. The only exception occurred among the elderly, which had lower HAI titers against the $\mathrm{CA} / 09 \mathrm{H} 1 \mathrm{~N} 1$ vaccine strain (Figure 2, B-E).

Young adults exhibit preexisting subtype immunodominance against IBV. Influenza virus infection has been associated with antigen and epitope immunodominance $(31,33)$. However, there are few reports detailing subtype immunodominance with tetravalent influenza vaccination. To evaluate the distribution of RB-Abs against each of the vaccine components prior to and following QIV, the percentage of total HAI activity in the serum was calculated. In the absence of preexisting immunodominance, we would expect similar HAI titers against each of the 4 strains in the vaccine that would account for about $25 \%$ of total serological HAI activity. However, prior to vaccination, serum HAI activity against the $\mathrm{B}_{\text {yam }}$ vaccine component was significantly higher than the other 3 vaccine components $(\mathrm{D} 0-25 \%)=4.17 \pm 0.42$ (Supplemental Figure 1A; supplemental material available online with this article; https://doi.org/10.1172/jci.insight.132155DS1). In contrast, serum HAI activity against the $\mathrm{B}_{\text {vic }}$ component was significantly lower (Supplemental Figure 1A). Furthermore, despite the significant impact of QIV to balance the HAI response to the 4 vaccine components $(P<0.0001)$, this was insufficient to overcome preexisting immunodominance against the $\mathrm{B}_{\text {yam }}$ strain (which remained $2.11 \% \pm 0.3 \%$ higher than the other components) (Supplemental Figure $1 \mathrm{~A}$ ). When stratifying by age, young adults had significant differences in HAI activity against the 2 influenza B strains prior to and following QIV (Supplemental Figure 1B). In contrast, HAI activity against each of the 4 vaccine strains was more balanced in the other age groups, particularly following vaccination (Supplemental Figure 1, C-E).

QIV induces HA-specific antibodies but cannot overcome preexisting subtype immunodominance. To assess the impact of QIV on total anti-HA binding antibody titers, we measured HA-specific IgG in the serum of healthy volunteers prior to and 21-28 days following vaccination. QIV elicited HA-specific antibodies against all 4 vaccine strains (mean D21-D0 = 104 $\pm 14 ; 40 \pm 5 ; 145 \pm 16$; and $115 \pm 13 \mu \mathrm{g} / \mathrm{mL}$ for H1N1, $\mathrm{H} 3 \mathrm{~N} 2, \mathrm{~B}_{\text {vic }}$, and $\mathrm{B}_{\text {yam }}$, respectively; Supplemental Figure $1 \mathrm{~F}$ ). After stratifying by age, only young adults (18-35 y.o.) had a significant increase in anti-HA antibody titers against both influenza A HA antigens following vaccination (Figure 3A). By days 21-28 after vaccination, HA-specific antibody titers were statistically similar across all vaccine strains and age groups, with the exception of $\mathrm{B}_{\text {yam }}$, which was significantly increased in young adults $(P=0.0014)$ (Figure $3, \mathrm{~A}-\mathrm{D})$. There was also a significant bias toward IBV antigens $(P<0.0001)$ and an extremely pronounced subdominant response against the H3N2 vaccine component $(P<0.0001)$ prior to and following QIV (Figure 3E). Interestingly, IBV immunodominance was particularly pronounced in subjects $18-50$ y.o. prior to and following vaccination (Figure $3, \mathrm{~F}$ and $\mathrm{G}$, and Supplemental Figure 1G). Middle-aged adults (50-65 y.o.) displayed antibody responses that were highly focused toward the $\mathrm{H} 1 \mathrm{~N} 1$ and $\mathrm{B}_{\text {vic }}$ vaccinal HA components before and after vaccination $(P<0.0001$, Fig- 
A

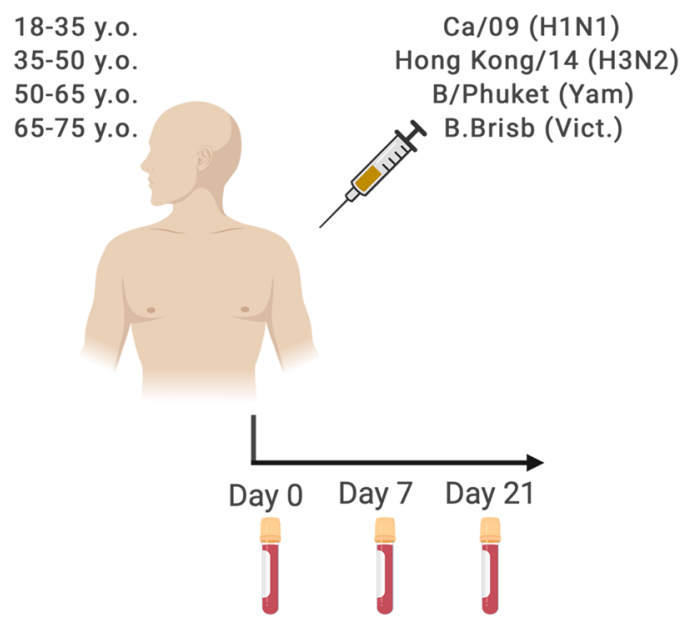

C

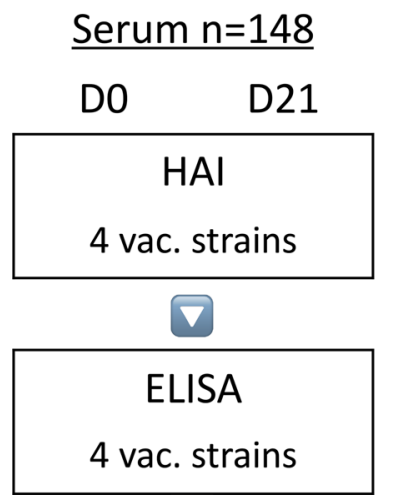

B Serum

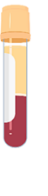

HAI

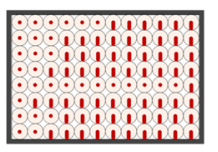

IgG ELISA

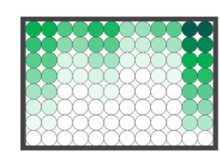

PBMCs $n=40$

DO

D21

In vitro Diff.

4 vac. strains
PBMC

FACS analysis

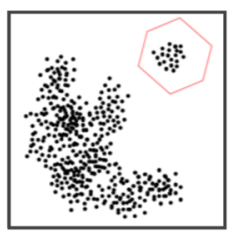

Plasmablast

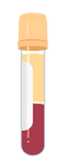

ELISpot

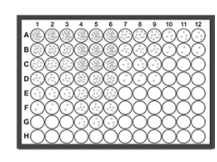

In vitro $\mathrm{B}_{\text {mem }}$ Diff.

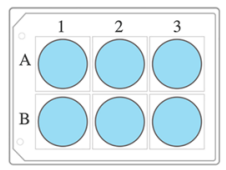

PBMCs $n=40$

D7

Flow Cytometry

IAV vac. strains

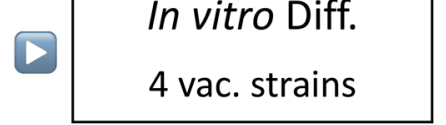

Representative sampling for age and sex. Elderly (65+) individuals excluded due to dosage differences

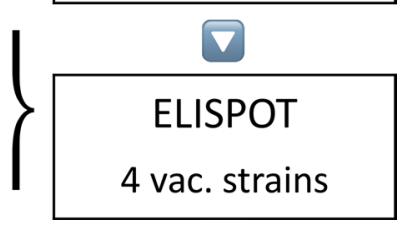

Figure 1. General experimental design. (A) Healthy volunteers were vaccinated with the standard dose (15 $\mu \mathrm{g} /$ antigen) split-virion (IIV) version of licensed Fluzone (Sanofi Pasteur), and serum and PBMCs samples were collected prior to, 7-9, and 21-28 days following vaccination. (B) Hemagglutination inhibition activity and total HA-specific IgC were measured in serum samples collected prior to and 21-28 days following vaccination, while frequency of plasmablast were quantified in peripheral blood 7-9 days following vaccination by flow cytometry and ELISpot. PBMC samples collected prior to and 21-28 days following vaccination were differentiated in vitro, and conditioned supernatants were tested for reactivity against the 4 vaccine components to quantify the memory-derived antibody response. (C) Sampling decision tree for each assay represented in $\mathbf{B}$.

ure $3 \mathrm{H}$ ). Of note, immunodominance against a particular strain after vaccination was positively correlated with preexisiting immunodominance prior to vaccination (Supplemental Table 1).

Increase in HA-specific IgG correlates with increased HAI titers after vaccination. To assess the impact of preexisting neutralizing antibody activity on anti-HA IgG titers following influenza vaccination, subjects were stratified by serological HAI activity against each vaccine strain prior to vaccination (Figure 4, A-D). Preexisting serological HAI activity did not impact anti-HA IgG titers following QIV (Figure 4, A-D). To understand the relationship between anti-HA IgG titers and HAI serological activity, we stratified our cohort by HAI activity after vaccination (Figure 4, E-L). Subjects with antibodies with high HAI activity at days 21-28 after vaccination had a significant increase in anti-HA IgG antibody titers to each component in the vaccine compared with prevaccination titers (Figure 4, E-H). Similarly, there was a large fold change in serum HAI activity that was associated with a significant increase in anti-HA IgG antibodies against the H1N1 and IBV vaccine strains (Figure 4, I-L). Furthermore, anti-HA binding antibody titers were positively correlated with HAI activity against each vaccine component prior to and following QIV (Supplemental Table 1).

Increased frequency of plasmablasts in peripheral blood is associated with enhanced serological HAI activity follow- 
A $18-75$ y.o.

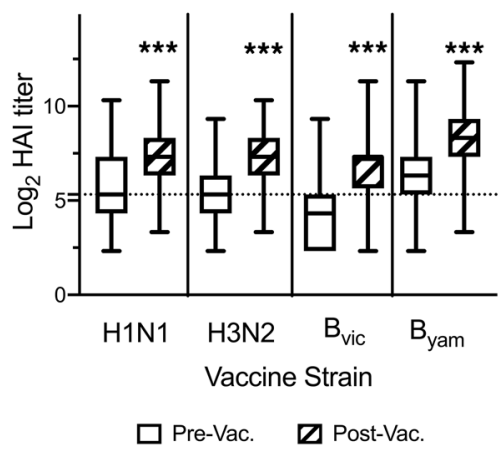

B

H1N1

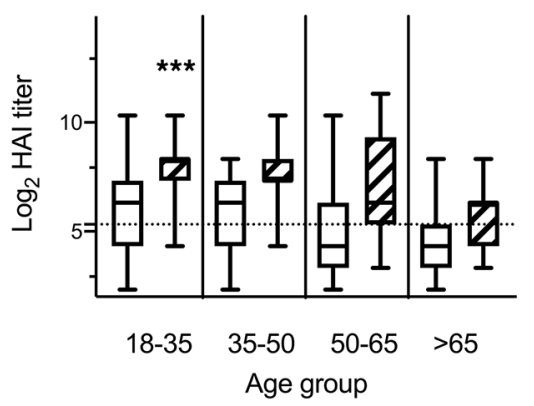

D B $_{\text {vic. }}$

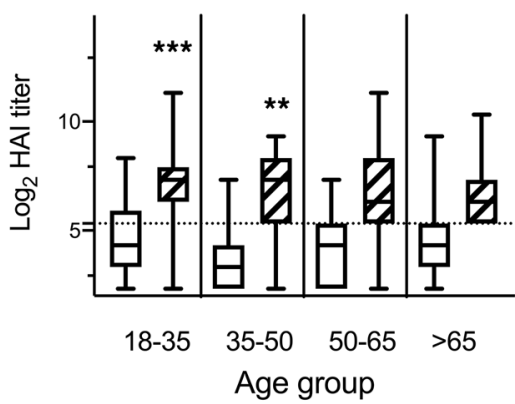

Pre-Vac.
C

\section{$\mathrm{H} 3 \mathrm{~N} 2$}

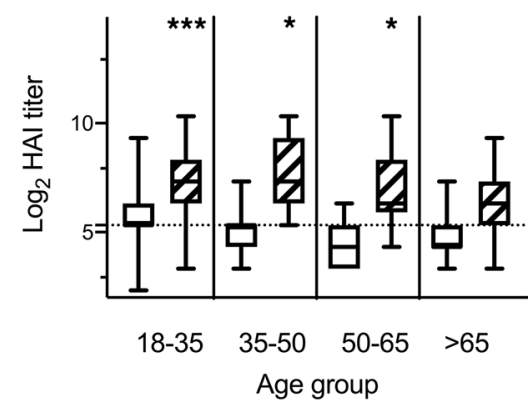

E $\quad B_{\text {yam. }}$

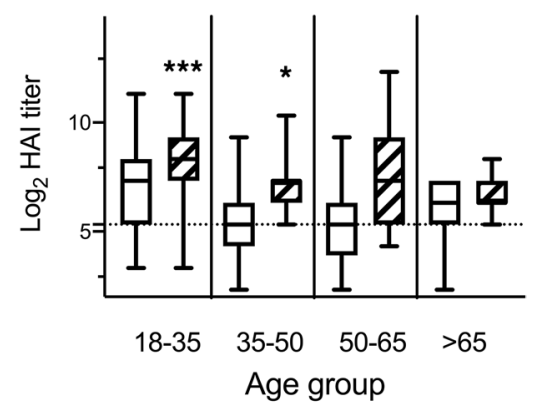

Post-Vac.

Figure 2. QIV elicits receptor blocking antibodies (RB-Abs) against all 4 vaccine strains. (A) Levels of RB-Abs in the serum of healthy volunteers (18-75 years old) prior to and 21-28 days following QIV were measured for hemaglutinaition inhibition (HAI) activity against each of the 4 vaccine strains. (B-E) HAl titer in healthy volunteers from different age groups against (B) A/Ca/09 H1N1 virus, (C) A/HK/14 H3N2 virus, (D) B/Brisb/08 Victoria-lineage virus, and (E) B/Phuket/13 Yamagata-lineage virus prior to and 21-28 days following QIV. ${ }^{*} P<0.05,{ }^{* *} P<0.01,{ }^{* * *} P<0.001$ by 1 -way ANOVA, Friedman test, and Dunn's multiple comparisons; $n=148$

ing vaccination. To assess whether the frequency of total plasmablasts (PB) (CD3/CD14- CD19+, CD27 $\mathrm{CD}^{2} 8^{++}$cells) present in peripheral blood at the peak of the immune response (D7) was associated with enhanced neutralizing antibody titers, we evaluated 40 subjects stratified according to their HAI activity against each vaccine strain using flow cytometry. As expected, the vast majority of $\mathrm{CD} 27^{+} / \mathrm{CD} 38^{++} \mathrm{B}$ cells are $\mathrm{IgD}^{-} / \mathrm{CD} 20^{-}$consistent with a $\mathrm{PB}$ phenotype (Figure 5A and Supplemental Figure 3A). Subjects with less than a 4-fold increase in HAI activity against all 4 vaccine strains (nonresponders) had a significantly lower number and frequency of PB than low-responders (4-fold increase in HAI titer to at least 1 of the vaccine components) or high-responders (an average 4-fold increase in HAI activity against all 4 vaccine components). Furthermore, the number and frequency of total PB was significantly elevated in high-responders compared with low-responders (Figure 5, B and C). Subjects with a $\geq 2$-fold increase in anti-HA IgG binding also exhibited a significantly higher number and frequency of total PB in peripheral blood 7 days after vaccination (Figure 5, D and $\mathrm{E}$ ). In contrast, once stratified, there was no difference in the number or frequency of total PB among the different age groups (18-34, 35-50, and 50-65 y.o.) (Figure 5, F and $\mathrm{G}$, and Supplemental Figure 2A).

Acute expansion of HA-specific plasmablasts is associated with increased serological HAI activity after vaccination. To understand the impact of preexisting immunity on the acute (day 7; D7) HA-specific PB response, the number of antibody-secreting cells (ASC) against each vaccinal HA component was quantified by ELISpot in 40 subjects with distinct prevaccination serological HAI profiles (Figure 6A). There was no difference in the HA-specific PB response against the 4 vaccine components in subjects $<65$ y.o. (Supplemental Figure 2, B and $\mathrm{C}$ ). Overall, the HA-specific response in vaccinated subjects accounts for $10 \%$ of the total $\mathrm{PB}$ response 7 days after vaccination and was comparable across the 4 vaccine strains (Supplemental Figure 2L). Moreover, after stratifying according to preexisting HAI titer (D0), there was no difference in the number of HA-specific ASC against each vaccine component between nonseroprotective $(\leq 1: 40 \mathrm{HAI}$ titer) or highly seroprotected 

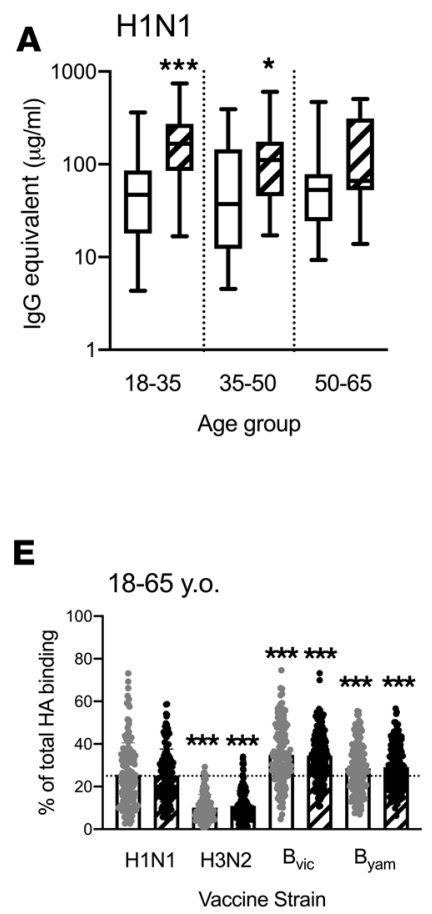
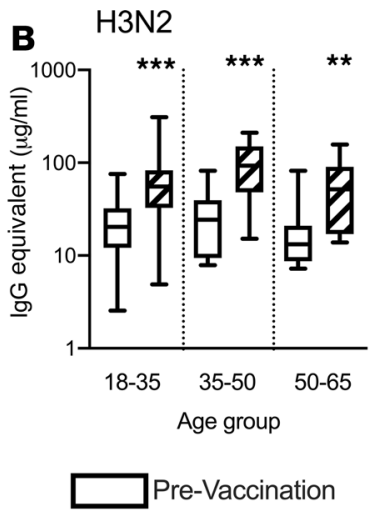

$\mathbf{F}$

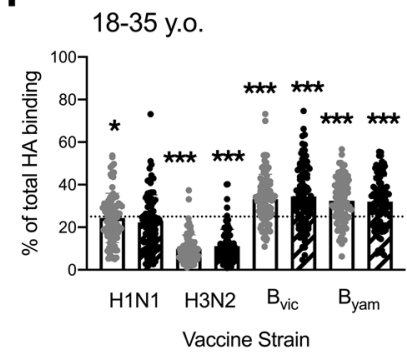

$\square$ Pre-Vaccination

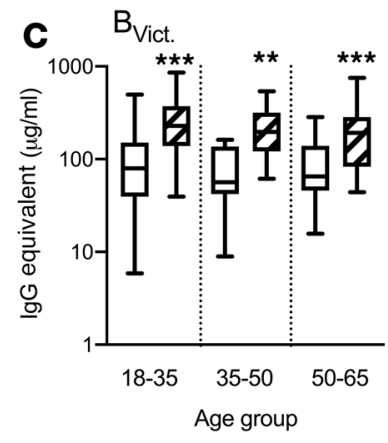

Z2 Post Vaccination

G

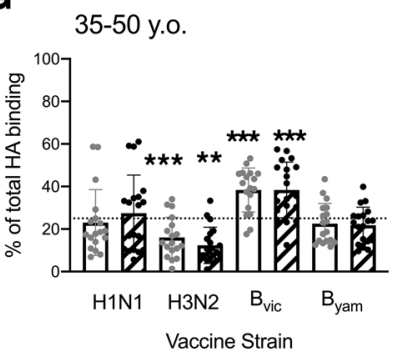

H

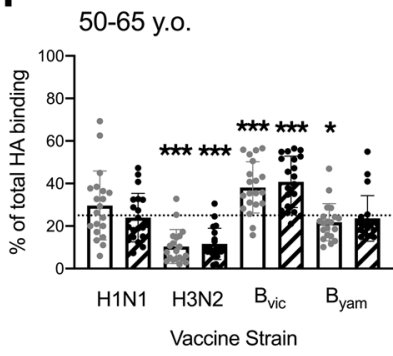

Figure 3. QIV increases HA-specific antibody titer against the $\mathbf{4}$ vaccine strains but cannot resolve preexisting subtype immunodominance. (A-D) HA-specific IgC levels in the serum of healthy volunteers from different age groups prior to and 21-28 days following QIV were measured by quantitative ELISA against the 4 vaccine strains: (A) A/Ca/09 H1N1 virus, (B) A/HK/14 H3N2 virus, (C) B/Brisb/08 Victoria-lineage virus, and (D) B/Phuket/13 Yamagata-lineage virus. (E) Percentage of HA binding to each vaccine strain from cumulative HA-reactive IgG response to QIV in healthy volunteers (18-65 y.0.) prior to and 21-28 days following vaccination. Horizontal dashed line represents the theoretical $25 \%$ response for each of the 4 vaccine strains in the absence of antigenic immunodominance. (F-H) Percentage of HA binding as in $\mathbf{E}$ stratified by age group; (F) 18-35 y.0. young adults, (C) 35-50 y.0. adults, and (H) 50-65 y.o. middle age. ${ }^{*} P<0.05,{ }^{* *} P<0.01,{ }^{* *} P<0.001$ by 1-way ANOVA, Friedman test, and Dunn's multiple comparisons; $n=148$

( $\geq 1: 80 \mathrm{HAI}$ titer) subjects (Figure 6B). In addition, individuals with high serological HAI titers 21-28 days following vaccination also displayed significantly increased numbers and frequencies of HA-specific ASC against each respective vaccine strain 7 days following vaccination (Figure 6C and Supplemental Figure 2, $\mathrm{D}-\mathrm{K})$. The percentage of total HA-specific ASC to each of the vaccine components was balanced between the $2 \mathrm{IBV}$ vaccine strains, but there was a significant bias to $\mathrm{H} 1 \mathrm{~N} 1$ vaccine strain compared with H3N2 (Figure 6D and Supplemental Figure 2M).

QIV strongly induces HA-specific memory B cell response. QIV recalls and expands preexisting HA-specific memory B cells $\left(\mathrm{B}_{\mathrm{mem}}\right)$. However, the immunodominant response against the H1N1 HA antigen in Fluzone cannot be attributed to differences in $\mathrm{HA}_{(\mathrm{H} 1)}$-specific $\mathrm{B}_{\text {mem }}$ frequency 7 days following vaccination (data not shown). To understand how vaccination reshapes the $\mathrm{B}_{\text {mem }}$ compartment, peripheral blood $\mathrm{B}$ cells from 40 subjects were subjected to in vitro differentiation, and the resulting antibody reactivity against the vaccine HA components in culture supernatants was assessed by ELISA. Across all age groups, preexisting $\mathrm{B}_{\text {mem }}$-derived antibody levels were low against the HA of each vaccine strain (Supplemental Figure 3, B-E). QIV significantly increased HA-specific, $\mathrm{B}_{\text {mem }}$-derived antibody levels against all 4 vaccine components following vaccination (Figure 7A). Furthermore, a substantial fold increase in the abundance of HA-specific $\mathrm{B}_{\text {mem }}$-derived antibody was commonly detected across all age groups, following vaccination with Fluzone (Supplemental Figure 3, $\mathrm{B}-\mathrm{E})$. In the case of $\mathrm{H} 3 \mathrm{~N} 2$ and $\mathrm{B}_{\text {yam }}$, a higher fold change in $\mathrm{B}_{\text {mem }}$-derived antibody response was associated with seroconversion (Figure 7, B, C, E, and F). When the percentage of $\mathrm{B}_{\text {mem }}$-derived antibodies that bound to each HA vaccine component was calculated, the preexisting memory response was well balanced across all 4 vaccine components. However, despite induction of $\mathrm{B}_{\mathrm{mem}}$ reactivity, there was clear subdominance in the $\mathrm{B}_{\text {mem }}$-derived antibody response against the H3N2 vaccine strain following QIV (Figure 7C).

$H 3 N 2$ vaccine cannot recall preexisting $B$ cell memory. To understand the prevalence of B cell memory immunodominance in this cohort, the number of individuals with a significantly increased abundance 


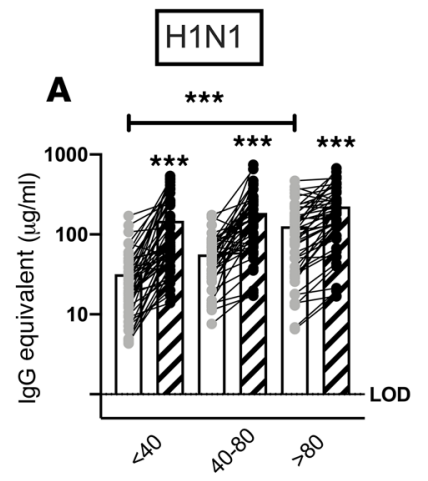

HAI Titer pre-vaccination

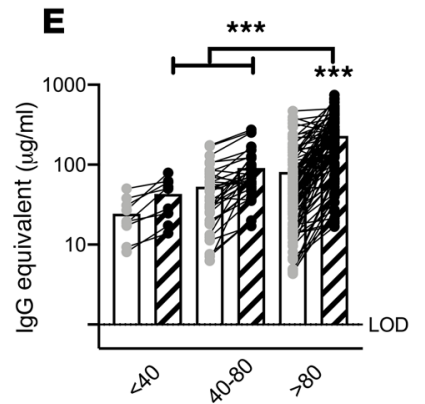

HAI Titer post-vaccination

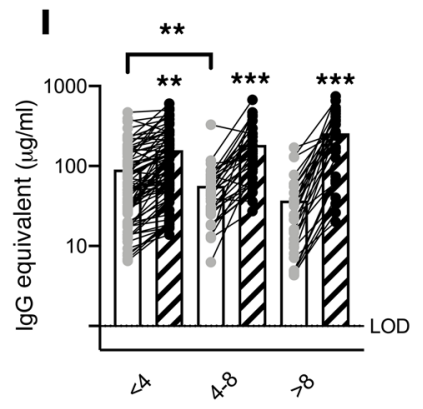

HAl Fold Change

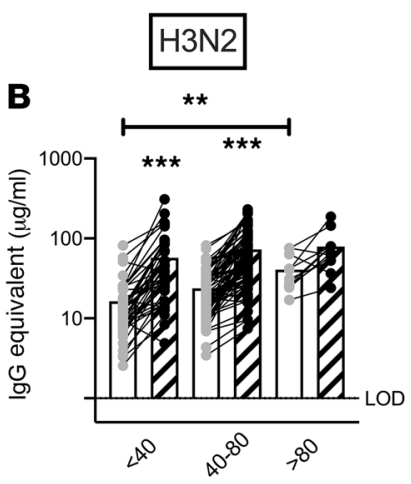

HAI Titer pre-vaccination

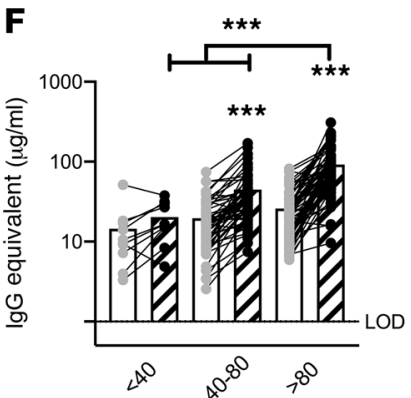

HAI Titer post-vaccination

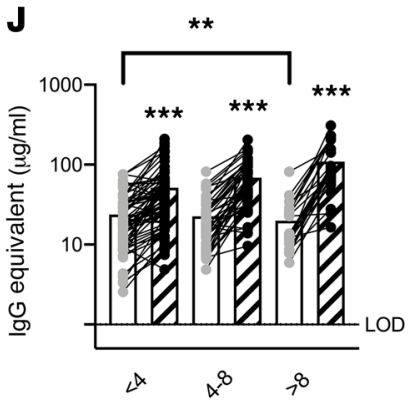

HAl Fold Change

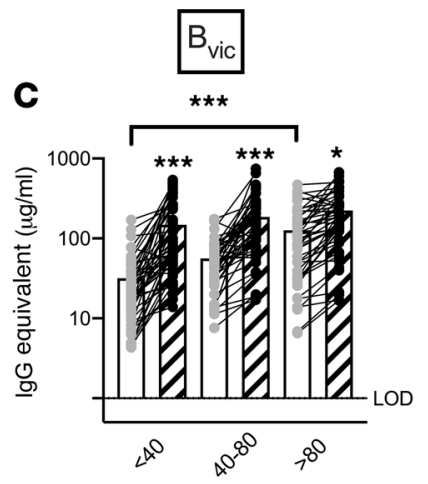

HAI Titer pre-vaccination

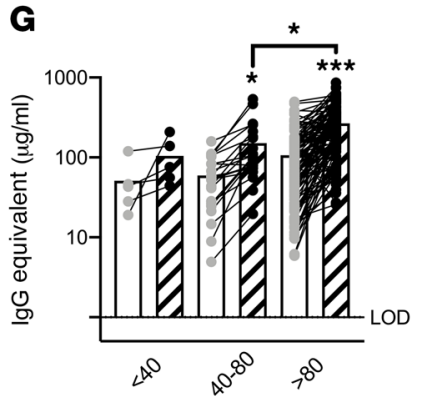

HAl Titer post-vaccination

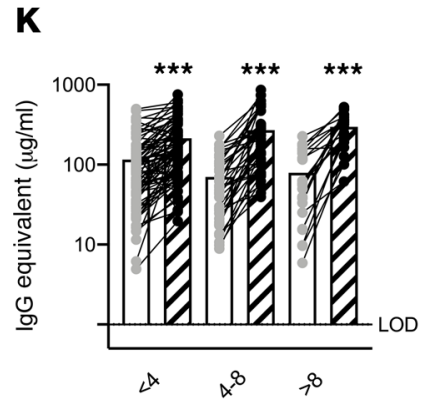

HAI Fold Change

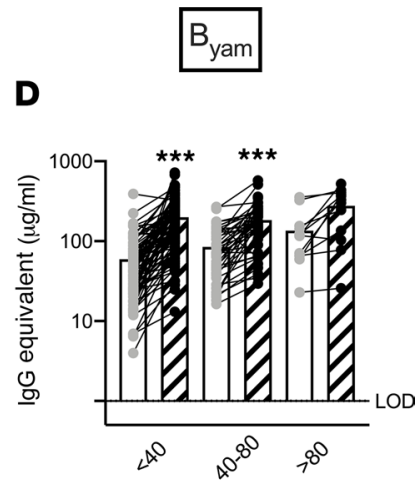

HAI Titer pre-vaccination

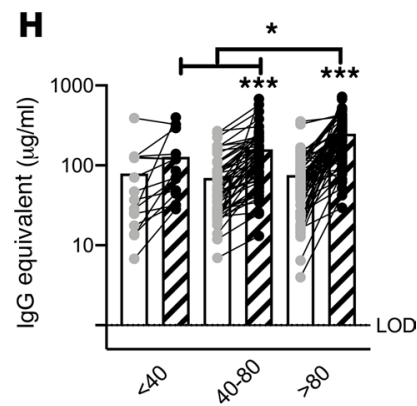

HAI Titer post-vaccination

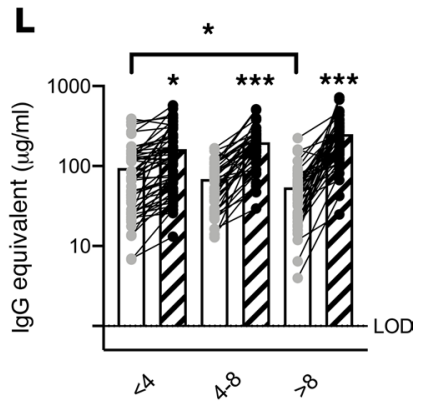

HAl Fold Change

HA-Specific IgG pre-vaccination

Dע HA-Specific IgG post-vaccination

Figure 4. Increased total HA-specific antibody is associated with high serological HAI activity. (A-D) HA-specific lgG antibody levels in the serum of healthy volunteers (18-65 y.o.) prior to and 21-28 days following QIV grouped by preexisting HAl titer to each of the vaccine strains. (E and F) HA-specific IgG antibody levels in the serum of healthy volunteers (18-65 y.o.) as in A-D but grouped by HAI titer 21-28 days after QIV to each of the vaccine strains. (I-L) HA-specific IgC antibody levels in the serum of healthy volunteers (18-65 y.o.) as in A-D but grouped but HAl titer fold change to each of the vaccine strains. A/Ca/09 H1N1 virus $(\mathbf{A}, \mathbf{E}$, and $\mathbf{I}), \mathrm{A} / \mathrm{HK} / 14 \mathrm{H} 3 \mathrm{~N} 2$ virus (B, F, and J), B/Brisb/08 (Victoria-lineage virus) (C, G, and $\mathbf{K})$, and B/Phuket/13 (Yamagata-lineage virus) (D, $\mathbf{H}$, and $\mathbf{L}){ }^{*} P<$ $0.05,{ }^{* *} P<0.01,{ }^{* *} P<0.001$ by 1 -way ANOVA, Friedman test, and Dunn's multiple comparisons; $n=148$

of $\mathrm{B}_{\text {mem }}$-derived antibodies that bound to at least 1 of the HA vaccine components was calculated. Surprisingly, prior to vaccination, more than $80 \%$ of subjects had a preferential $\mathrm{B}_{\text {mem }}$ response to at least 1 of the vaccine components. After vaccination, all subjects had a preferential response to at least 1 of the vaccine components (Figure $8 \mathrm{~A}$ ). A similar number of individuals had an immunodominant $\mathrm{B}_{\text {mem }}$ response to each vaccine component prior to vaccination. Following QIV, fewer subjects had an immunodominant response to the H3N2 component (Figure 8, B-D). When stratifying by age, there was a strong memory immunodominance against the 2 IAVs in young adults (18-35 y.o.) prior to vaccination, but there were no subjects in this age group with an $\mathrm{H} 3 \mathrm{~N} 2$ immunodominant memory response following vaccination (Figure $8, \mathrm{E}-\mathrm{G}$ ).

QIV induces stem-directed B cell memory. To understand the impact of QIV in the memory response 
A

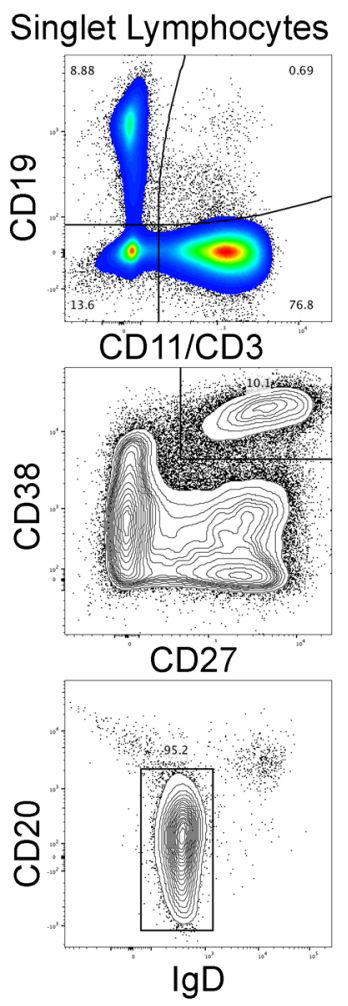

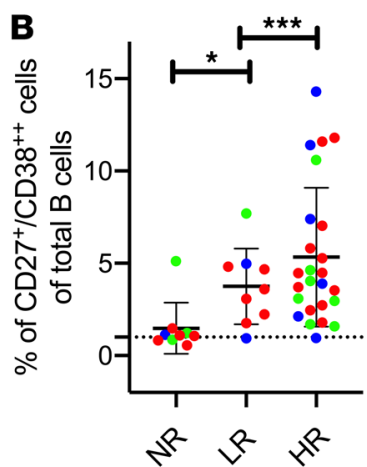

HAI Group

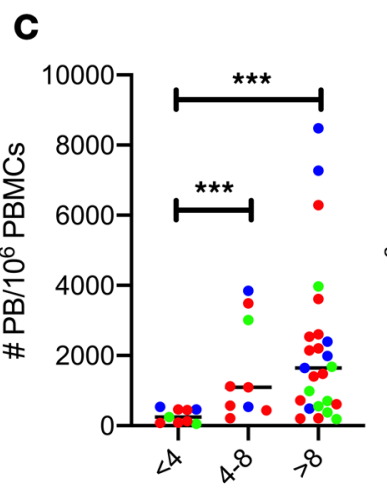

HAl Fold Change

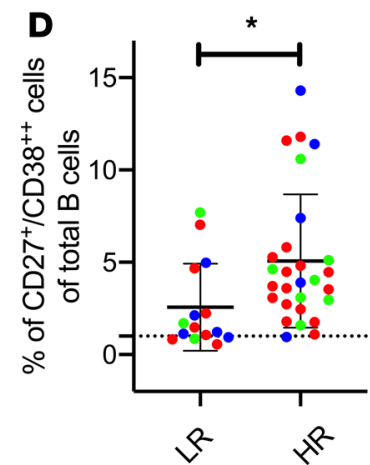

IgG Fold increase

\section{E}

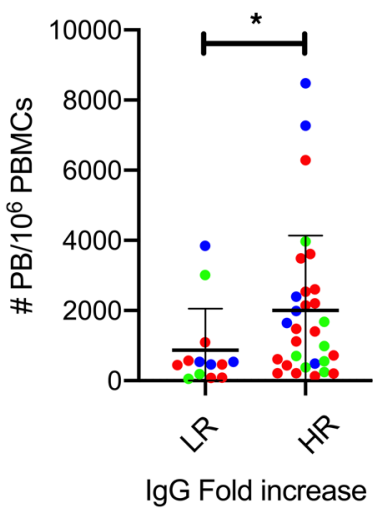

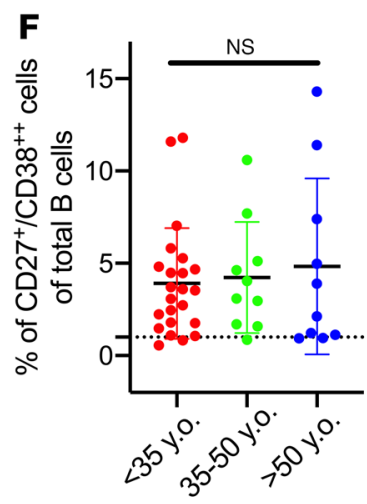

G

Age group

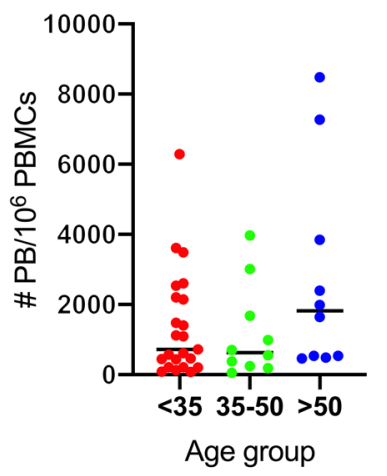

$18-35$ y.o. $35-50$ y. 0 $50-65$ y.o

Figure 5. Peripheral blood plasmablast (PB) response 7 days after QIV is associated with serological HAI activity. (A) Representative gating strategy for quantification of PB in peripheral blood mononuclear cells (PBMC) from healthy volunteers 7 days after QIV. (B and C) Summary data for frequency (B) and total number (C) of PB, measured as in $\mathbf{A}$, in healthy volunteers categorized as nonresponders (NR), low responders (LR), or high responders (HR) to QIV vaccine. (D and E) Summary data for frequency (D) and total number (E) of PB in healthy volunteers categorized as LR and HR by total HA-specific IgG response to QIV vaccine. (F and $\mathbf{G})$ Summary data for frequency $(\mathbf{F})$ and total number $(\mathbf{G})$ of PB in healthy volunteers from different age groups. ${ }^{*} P<0.05,{ }^{* *} P<0.001$ by 1 -way ANOVA, Friedman test, and Dunn's multiple comparisons; $n=40$

to the HA-stem region of H1N1 and H3N2 IAV, we quantified the levels of $\mathrm{B}_{\text {mem }}$-derived antibodies against chimeric $\mathrm{cH} 6 / 1$ and $\mathrm{cH} 7 / 3$ recombinant $\mathrm{HA}(\mathrm{rHA})$ probes prior to and following vaccination. $\mathrm{B}_{\text {mem }}$-derived antibody titers directed against the HA stem region were significantly increased in all age groups after vaccination (Figure 9, A and B). No difference between group $1(\mathrm{cH} 6 / 1)$ and group 2 (cH7/3) anti-stem titers were detected prior to vaccination. However, $\mathrm{B}_{\text {mem }}$-derived anti-H1 HA stem antibodies were significantly increased relative to anti-H3 HA stem titers following QIV (Supplemental Figure 3, $\mathrm{F}$ and $\mathrm{G}$ ). Despite increases in $\mathrm{B}_{\text {mem }}$-derived antibody reactivity after vaccination, there was a general trend for a decrease in the relative percentage of $\mathrm{B}_{\mathrm{mem}}$-derived stem antibodies overall (Supplemental Figure $3 \mathrm{H}$ ). Nevertheless, there was no significant difference in the fold change of antibodies that bond to the chimeric $\mathrm{cH} 6 / 1$ and $\mathrm{cH} 7 / 3 \mathrm{rHA}$ and the respective $\mathrm{H} 1 \mathrm{~N} 1$ or $\mathrm{H} 3 \mathrm{~N} 2 \mathrm{HA}$ vaccine components following QIV (Figure 9, C and D).

\section{Discussion}

The WHO recommends yearly vaccination as the primary strategy to prevent influenza virus infection and transmission. Multivalent, inactivated influenza vaccines (IIV) represent over $90 \%$ of global influenza vaccine market share and are expected to dominate the market until 2022 (45). Influenza activity during the 2016-2017 Northern hemisphere influenza season was moderate across all age groups, but there was increased spread of H3N2 IAVs from December to mid-March, followed by an increased number of IBV infections over the last 2 months of the season $(46,47)$. Over the past 40 years, H3N2 IAVs have been the predominant seasonal influenza subtype in more than half the influenza seasons $(23$ of 40$)(46,48)$. In contrast, H1N1 influenza virus infections only surpassed H3N2 influenza virus infections in 10 of the last 
A

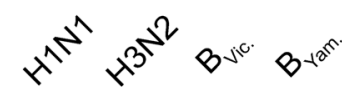

Pt\# 51

$\mathrm{Pt} 73$

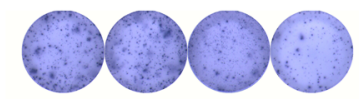

Pt\# 35

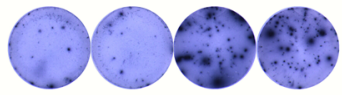

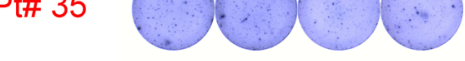

Pt\# 42
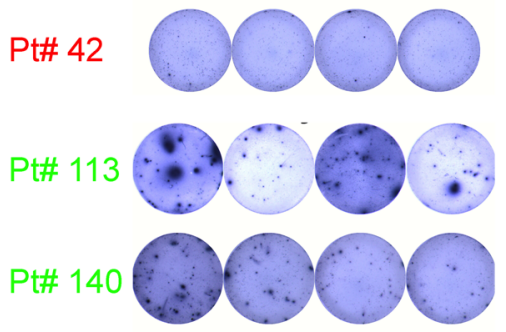

Pt\# 21

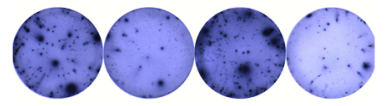

Pt\# 8

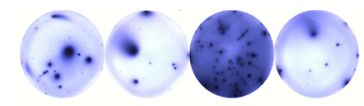

Pt\# 130

Pt\# 86
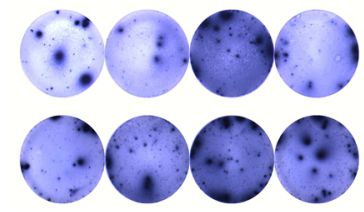
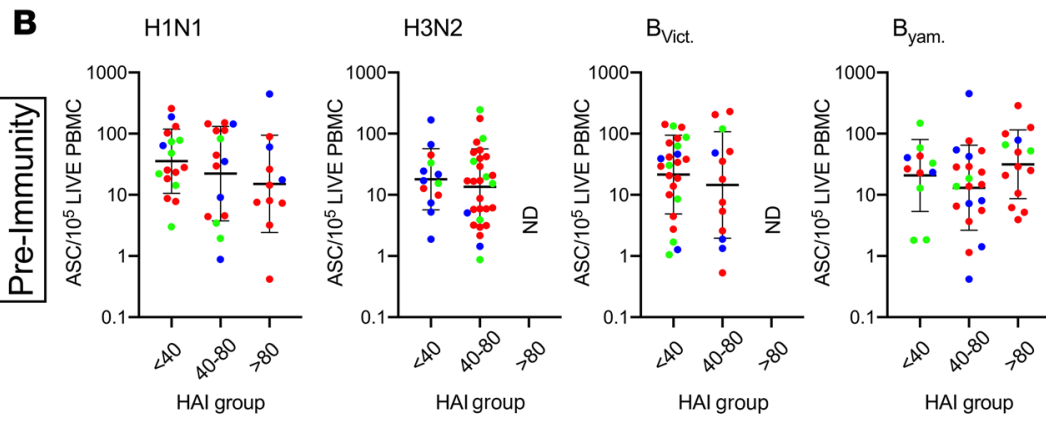

18-35 y.o. $35-50$ y.o. 50.65 y.o.
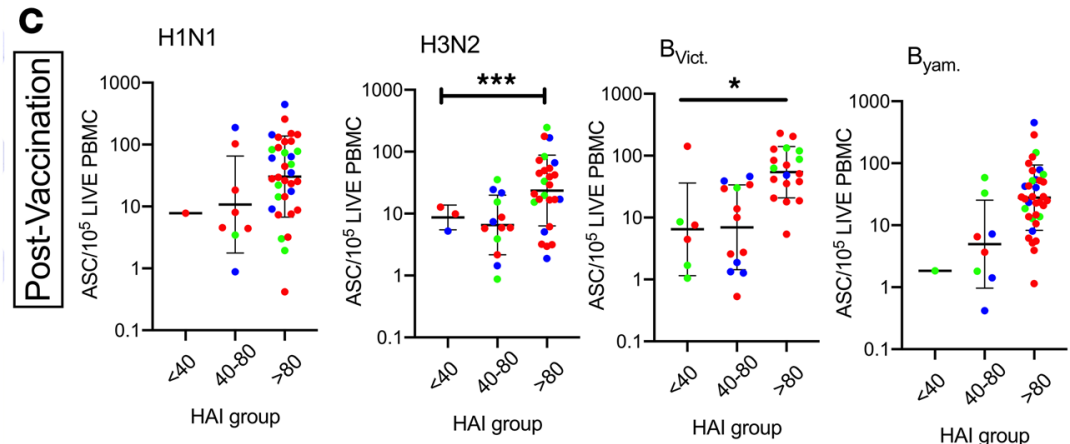

18-35 y.o. $35-50$ y.o 50.65 y.o.

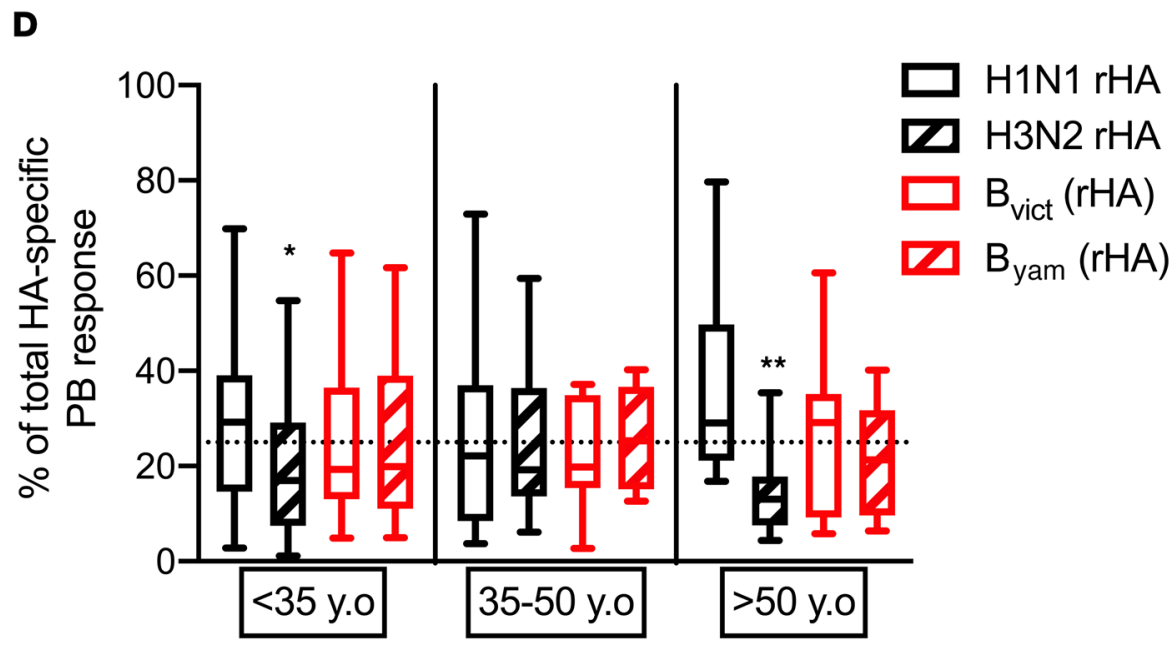

Figure 6. QIV elicits subdominant plasmablast response to the H3N2 influenza vaccine strain. (A) Representative ELISpot with day 7 PBMCs from 10 individuals of varying age and response to the 4 vaccine strains. (B and C) Number of HA-specific antibody secreting cells (ASC) against each vaccine component, quantified as in $\mathbf{A}$ per $1 \times 10^{5}$ PBMC, from 40 donors stratified by their serological HAl response prior to (B) and 21-28 days following (C) vaccination. PBMC samples were collected 7 days after vaccination. (D) Percentage of ASC to each vaccine strain, quantified as in $\mathbf{A}$ and normalized to total vaccine ASC response, in 40 donors stratified by age group. ${ }^{*} P<0.05,{ }^{* *} P<0.01,{ }^{* *} P<0.001$ by 1 -way ANOVA, Friedman test, and Dunn's multiple comparisons; $n=40$

42 seasons: 6 before and 4 after the 2009 pandemic $(46,48)$.

Influenza VE is highly variable between seasons, subtypes, and age groups $(41,44,49,50)$. Over the past 10 years, H3N2 influenza VE is consistently below $50 \%$ and lower than the corresponding H1N1 influenza A subtype vaccine or influenza B vaccine strains (9). During the 2016-2017 season, overall VE was approximately $40 \%$ against all subtypes and across all age groups. VE against $\mathrm{H} 3 \mathrm{~N} 2$ viruses across all ages was approximately $33 \%$, showing increased efficacy in young children (0.5-8 y.o. children, $50 \%)$ and the middle aged (50-64 y.o., 30\%), and lowest in young adults (18-49 y.o., $13 \%$ ) and elderly ( $>65$ y.o., $21 \%)$ (51). The reason behind such striking differences in vaccine efficacy against the H3N2 influenza A subtype 


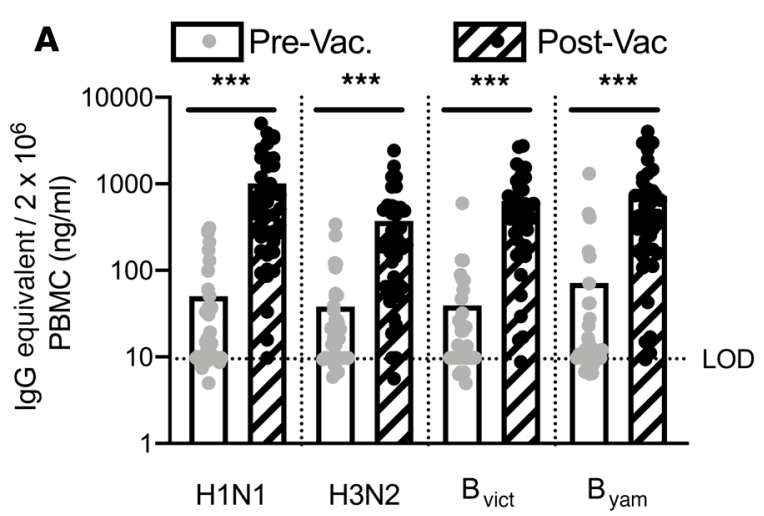

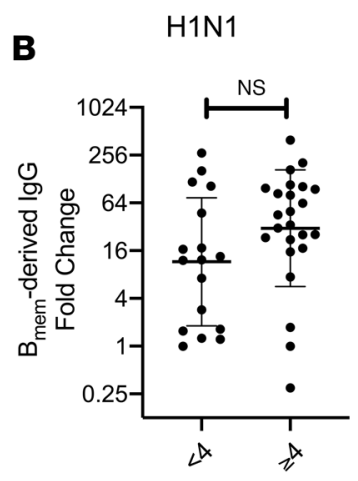

HAl Fold Change

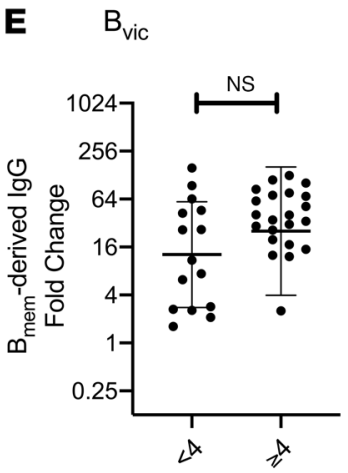

HAl Fold Change

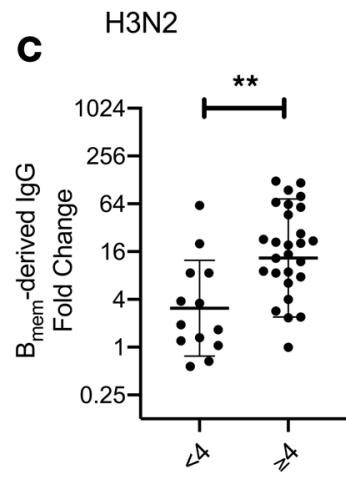

HAI Fold Change

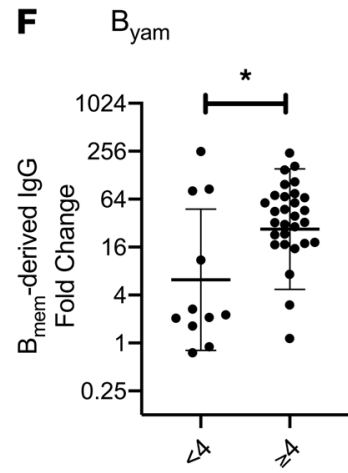

HAI Fold Change

Figure 7. QIV strongly induces HA-specific memory B cell responses. (A) Levels of memory B cell-derived ( $B_{\text {mem }}$-derived) antibody (IgG) against the 4 vaccine strains prior to and 21-28 days following QIV measured by ELISA following in vitro differentiation of PBMC from healthy volunteers (18-65 years old). (B, C, E, and F) Fold change following vaccination, in levels of $\mathrm{HA}$-specific $\mathrm{B}_{\text {mem }}$-derived IgG against each vaccine component, measured as in $\mathbf{A}$, in 40 donors categorized by $\mathrm{HAl}$ response to the corresponding vaccine strain. (D) Frequency of HA-specific $B_{\text {mem }}$-derived IgG to each strain of the total vaccine response prior to (left) and 21-28 days following (right) QIV, measured as in $\mathbf{A} .{ }^{*} P<0.05,{ }^{* *} P<0.01$, ${ }^{* * *} P<0.001$ by 1-way ANOVA, Friedman test, and Dunn's multiple comparisons; $n=40$

virus remains elusive and is of utmost importance in the search for a truly universal influenza vaccine.

Here, we thoroughly characterized the immune response to QIV in 18-65 y.o. adults. In accordance with previous reports (49), QIV strongly increases HA-specific and RB-Abs in the serum 21-28 days following vaccination (Figure 1). However, the magnitude of the response to each vaccine component was highly variable between individuals, either as a consequence of initial serological titers (13) or immunological dominance to one of the vaccine components (32). Serological subtype immunodominance is more pronounced in response to IBVs and seems to be exacerbated in 18-35 y.o. individuals (Figure 2 and Figure 3). Surprisingly, total HA-specific IgG binding activity was increased against the $B_{\text {vic }}$ vaccine strain. However, our data reveal that HAI activity was preferentially directed against the $B_{\text {yam }}$ IBV vaccine strain (Figure 3 and Supplemental Figure 1). This discrepancy might result from the artificial nature of the HAI assay, which - in the case of IBVs requires ether pretreatment for increased viral HA activity (43). It is plausible that ether extraction exposes conserved immunodominant epitopes in the $\mathrm{B}_{\text {yam }}$ strain that are otherwise inaccessible on the surface of the native protein $(52,53)$. Alternatively, these differences in immunodominance of RB-Abs versus total HA-specific IgG levels could be explained by the higher mutation rate and antigenic diversity of $\mathrm{B}_{\text {vic }}$ viruses $(54,55)$. In fact, this hypothesis seems to be better supported by the strong correlation between HAI serologic activity and total HA-specific antibody titers to both IBVs (Supplemental Table 1). Future studies should further elucidate these phenomena by comparing the serological antibody response with ether-extracted and native rHA proteins.

Epitope immunodominance in the HA protein has been extensively reported (56-61). In contrast, subtype immunodominance during polyvalent influenza vaccination has not been experimentally addressed. In this report, more than $90 \%$ of 18-65 y.o. adults had an immunodominant response to one of the vaccine strains that could not be overcome by vaccination. Initial reports described significantly reduced efficacy of polyvalent influenza vaccines compared with matched monovalent versions $(23,24)$. At the time, these differences were attributed to antigen quantity between the polyvalent and monovalent formulations (23). 
A

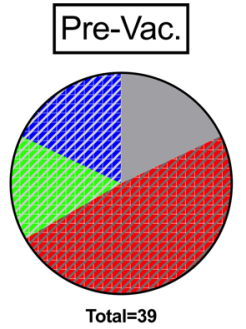

B Against all vac. strains

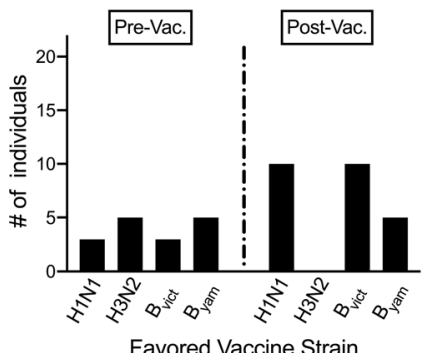

E $18-35$ y.o.

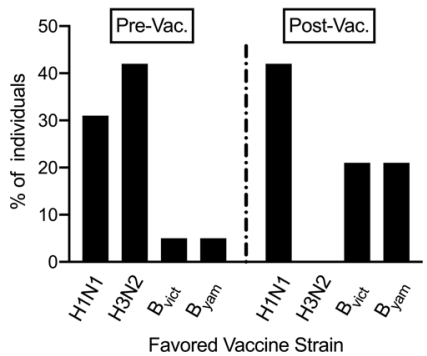

\section{Post-Vac.}

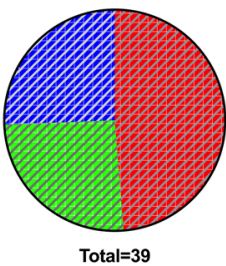

C Against IAV strains

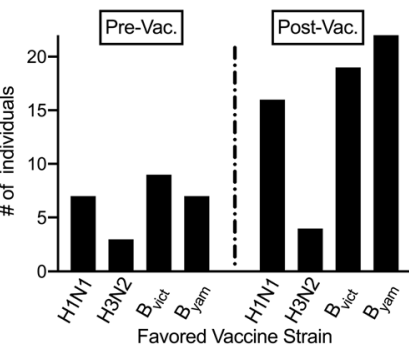

F $\quad 35-50$ y.o.

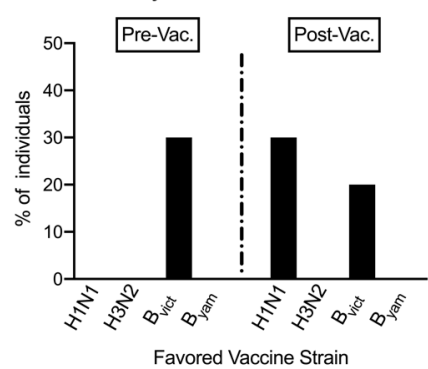

No-immunodominance

Immunodominance (18-35 y.o)

Immunodominance (35-50 y.o)

Immunodominance (50-65 y.o)

D Against IBV Strains

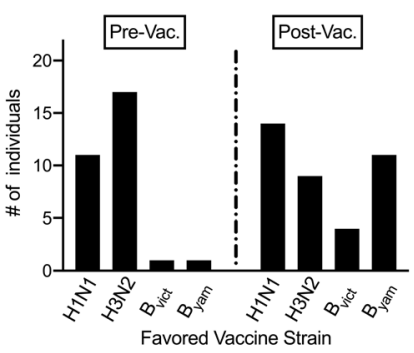

G

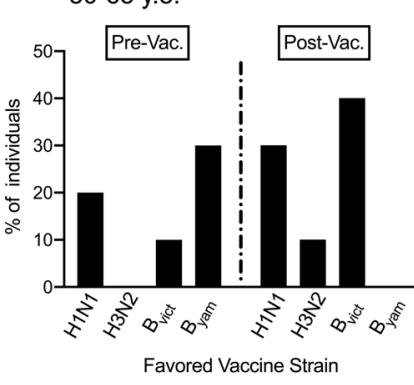

Figure 8. Memory B cell-derived antibody response to H3N2 vaccine strain is decreased compared with the other 3 QIV vaccine strains. (A) Proportion of individuals with a significantly increased memory B cell-derived ( $B_{\text {mem }}$-derived) antibody response to at least 1 of the vaccine strains prior and 21-28 days following vaccination. Colors denote donors within each age group. (B-D) Number of volunteers with a significantly higher $\mathbf{B}_{\text {mem }}$-derived antibody response to each of the vaccine strains compared with all other vaccine strains (B), only Influenza-A $(\mathbf{C})$, or only influenza-B vaccine (D) strains prior to and 21-28 days following QIV. (E-C) Percentage of subjects within different age groups with a significantly higher $\mathrm{B}_{\text {mem }}$-derived antibody response to each of the vaccine strains compared with all other vaccine strains $(\mathbf{E})$, only Influenza-A (F), or only influenza-B (G) vaccine strains prior and 21 days after QIV.

However, in light of our findings, we speculate that these differences, instead, might have arisen due to underlying immunodominance against one of the vaccine components as a result of preexisting immunity.

For decades, vaccine-elicited immune protection against influenza virus has been measured by the gold standard hemagglutination inhibition assay (62). However, recent studies indicate that alternative biomarkers correlate and may more accurately portray the immune response to influenza vaccination, as well as monitor effector functions that significantly reduce disease severity (63-68). Vaccine-induced HA-specific IgG increases and plasmablast expansion positively correlated with HAI seroprotection to each vaccine strain 21-28 days following vaccination (Figure 5, Figure 6, and Figure 7). Furthermore, the magnitude of HA-specific IgG response and plasmablast expansion were comparable among subjects ranging from 18-65 y.o. Collectively, our assessment of the immunological response elicited by QIV indicated that it was quite similar across the different age groups (18-65 y.o.).

The contribution of early-life influenza infection (imprinting) and subsequent influenza infection history (preimmunity) in the response to influenza vaccination is still controversial $(35,40,69,70)$. Imprinting seems to shape the long-lived subtype-specific antibody response in the serum, but subsequent influenza infections can determine the magnitude of these responses (39). There appears to be a similar bias between subtypes after vaccination with QIV. Individuals born in the 1950s to the mid-1960s, a time when only group 1 (H1N1 or H2N2) influenza viruses circulated in the human population, had the largest bias toward the H1N1 vaccine 
A
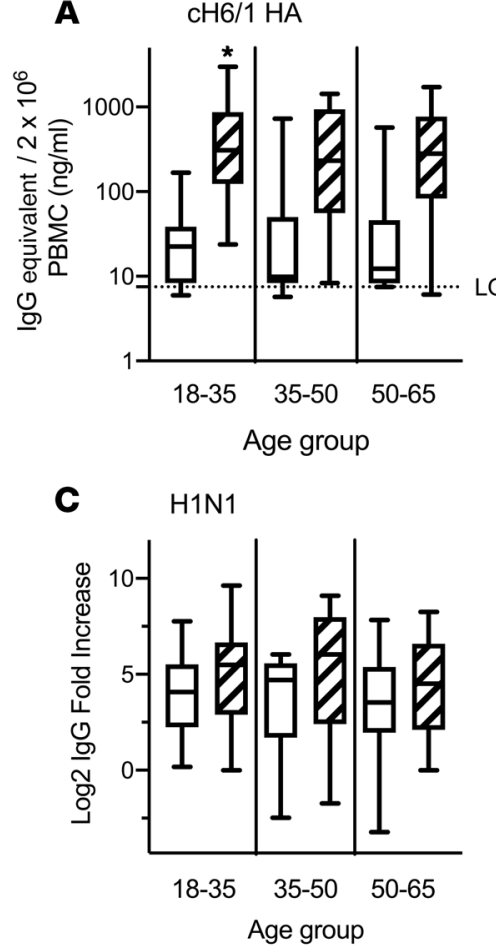

B

$\mathrm{cH} 7 / 3 \mathrm{HA}$

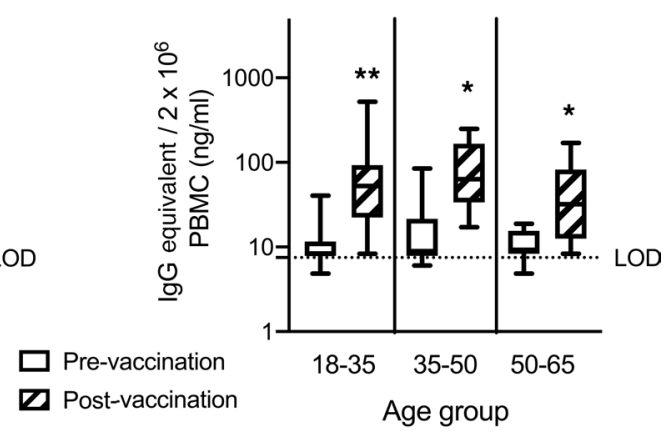

D $\quad \mathrm{H} 3 \mathrm{~N} 2$

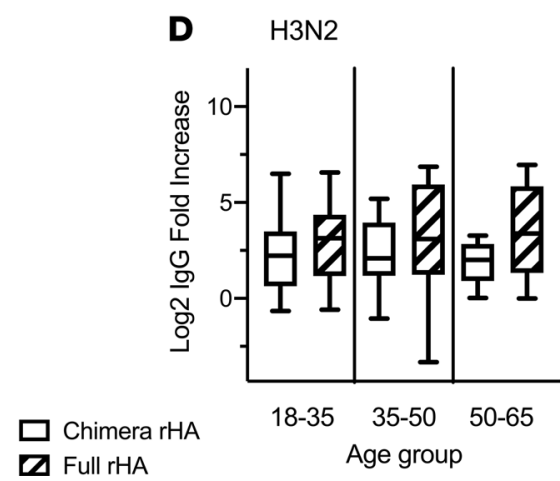

Figure 9. QIV induces stem reactive B cell memory. (A and B) Levels of $\mathrm{cH6} / 1$-reactive (A) or $\mathrm{CH7/3-reactive} \mathrm{(B)} \mathrm{memory}$ $B$ cell-derived ( $B_{\text {mem }}$-derived) antibody prior to and 21-28 days following vaccination, after in vitro differentiation. (C and D) Fold change following vaccination, in $\mathrm{HA}_{\text {stem }}$-directed and full HA-binding $\mathrm{B}_{\text {mem }}$ antibodies to H1N1 (C) or H3N2 (D) vaccine strains, in 40 subjects of varying age, measured as in $\mathbf{A}$ and $\mathbf{B}$. ${ }^{*} P<0.05,{ }^{* *} P<0.01$ by 1 -way ANOVA, Friedman test, and Dunn's multiple comparisons; $n=40$.

component. In contrast, younger subjects who were born when both H1N1 and H3N2 viruses circulated had a more balanced response to both IAV subtypes prior to vaccination (Figure 3). Our data are in agreement with previously published work by Nachbagauer et al. (39) where experienced subjects (49-64 y.o.) showed increased serum antibodies to group 1 influenza viruses relative to younger individuals.

It has been theorized that high levels of preexisting, cross-reactive antibodies can reduce the vaccine antigen dosage through absorption and clearance, ultimately leading to a decreased immune response (71). Although this phenomenon might be true when comparing the response to homosubtypic viruses, our data did not reveal a negative impact of increased preexisting antibody levels on the response to the respective vaccine strain components (Figure 5).

Influenza virus infection generates a strong and long-lasting $\mathrm{T}$ cell-dependent $\mathrm{B}_{\mathrm{mem}}$ response (42). In contrast, nonadjuvanted IIV rely on preexisting B cell memory (72). In our cohort, we observed a 15- to 30-fold increase in the HA-specific $\mathrm{B}_{\text {mem }}$-derived antibody response after vaccination that positively associated with the induction of serum HAI activity (Figure 7B). Furthermore, the 2016-2017 H3N2 vaccine component generated a significantly subdominant $\mathrm{B}_{\mathrm{mem}}$ response relative to the other vaccine strain components. Together, these results corroborate the importance of preexisting B cell memory against each influenza vaccine strain component in the overall response elicited by seasonal influenza vaccination.

Broadly reactive antibodies directed to the conserved stem portion of the HA protein can protect against heterosubtypic IAV $(19,73,74)$. However, the highly variable head portion of the HA protein is profoundly immunodominant during influenza vaccination, and it facilitates viral escape (17). Thus, recurrent vaccination may lead to antigenic focusing toward globular head, strain-specific immunodominant epitopes and consequently decrease broadly reactive stem-directed antibody responses $(21,75)$. Here, we observed that QIV increased $\mathrm{B}_{\text {mem }}$-derived stem-directed antibodies across all age groups. However, the magnitude of the stem-directed response was lower than that directed toward the HA globular head. Furthermore, the $\mathrm{B}_{\text {mem }}$-derived antibody response against the group 2 (H3N2) stem region was significantly subdominant compared with the response directed against the group 1 (H1N1) vaccine strain (Figure 9). 
This study highlights the importance of immunoprofiling and systematic characterization of the immune response to influenza vaccines. Gold standard serological assays alone, in the absence of corresponding cellular analysis, would fail to detect these significant differences in immunogenicity by the H3N2 strain compared with the other vaccine components. Overall, this study describes subtype immunodominance during QIV that resulted in a pronounced subdominant response to the H3N2 vaccine strain. The mechanism driving such striking differences in the magnitude of the serological and $\mathrm{B}_{\text {mem }}$ response to H1N1 and H3N2 vaccine strains is unclear. The 2016-2017 H3N2 vaccine strain was a good match to the majority of circulating H3N2 IAV and was maintained for the subsequent 2017-2018 Northern hemisphere season. Nonetheless, we observed mild influenza activity during the 2016-2017 season and severe influenza activity during the 2017-2018 season, both dominated by H3N2 influenza viruses. A recent report showed that $\mathrm{H} 3 \mathrm{~N} 2$ circulating viruses contained a K160T mutation on a glycosylation site in antigenic site B that was absent in the egg-adapted vaccine strain (76). It is possible that glycosylation changes in this antigenic site had a significant impact on $\mathrm{B}_{\mathrm{mem}}$ recall and consequently decreased immunogenicity. Alternatively, frequent changes in the $\mathrm{H} 3 \mathrm{~N} 2$ vaccine strain, while maintaining the same $\mathrm{H} 1 \mathrm{~N} 1$ vaccine component, might favor the response to repeated antigens through building upon the observed immunodominance. Unfortunately, this cross-sectional study does not allow us to address the mechanisms driving subtype immunodominance. Future longitudinal studies will clarify the impact of repeated seasonal influenza vaccination, with a focus on the development and maintenance of B cell memory and the influence of vaccine strain changes on the overall response elicited by polyvalent influenza vaccines.

\section{Methods}

Subjects and vaccine. Eligible subjects between the ages of 18 and 65 y.o., who had not yet received the seasonal influenza vaccine, were enrolled beginning in September of 2016. Vaccine formulation was based on WHO recommendations for the Northern hemisphere influenza seasons beginning in the fall, and as such, all vaccinations and collections occurred between November 2016 and March 2017. Subjects were vaccinated with the standard dose (15 $\mu \mathrm{g}$ /antigen) split-virion (IIV) version of licensed Fluzone (Sanofi Pasteur). The IIV formulation consisted of 4 strains of influenza strains in accordance with recommendations from the WHO and included for the 2016-2017 season: A/California/7/2009 (H1N1), A/Hong Kong/4801/2014 (H3N2), B/Phuket/3073/2013 (Yamagata-lineage), and B/Brisbane/60/2008 (Victoria-lineage).

During the 2016-2017 season, 148 eligible subjects were enrolled (Table 1). Blood (70-90 mL) was collected from each subject at the time of vaccination (D0) and collected again 7-9 days (D7) and 21-28 days (D21) after vaccination in order to facilitate subject compliance and recruitment. Blood samples were processed for sera and PBMC. For PBMC isolation, blood was collected in Vacutainer CPT tubes (BD Biosciences) at D0, D7, and D21. These samples were processed within 6 hours of collection and stored at liquid nitrogen (vapor phase) for future analysis. Serum was collected in Vacutainer SST tubes (BD Biosciences) and processed within 24-48 hours, stored at $4^{\circ} \mathrm{C}$ until separated, and aliquoted for long-term storage at $-20^{\circ} \mathrm{C}$. Overall workflow for each sample and time point, as well as selection criteria for each assay, is summarized in Figure 1.

Viruses and HA antigens. Influenza viruses were obtained through the Influenza Reagents Resource (IRR), BEI Resources, or the Centers for Disease Control and Prevention (CDC) or they were provided by Sanofi Pasteur and Virapur LLC. Viruses were passaged once in the same growth conditions as they were received, in 10-day-old embryonated, specific pathogen-free (SPF) chicken eggs per the protocol provided by the WHO. Due to low IBV sensitivity in the HAI test, viruses underwent ether treatment as recommended by the influenza division of the CDC in order to increase assay sensitivity, and more reliable detection of HAI rises following influenza B vaccination (52). Ether-extracted split viruses were created from freshly harvested allantoic fluid and from previously frozen virus lots, prior to HA and HAI assays, as previously described (77). Briefly, viruses were mixed at a 1:1 ratio with anhydrous diethyl ether (ACROS Organics, Thermo Fisher Scientific) for $\geq 4$ hours, with stirring. Following phase separation, ether was evaporated under a fume hood. Titrations before and after ether treatment were performed with turkey erythrocytes, and virus was standardized to $8 \mathrm{HAU} / 50 \mu \mathrm{L}$ for use in HAI assays. The virus used in this study matched the 4 vaccine strains included in the 2016 commercially licensed split-virion (IIV) Fluzone.

$r H A$ proteins. Full-length HA proteins were developed for each of the Fluozone 2016/17 quadrivalent vaccine components: H1N1, H3N2, Victoria-lineage, and Yamagata-lineage. Chimeric HA proteins were generated with the globular head portion of $\mathrm{A} /$ mallard/Sweden/81/2002 (A/H6N2) in the context of A/ California/07/2009 (A/H1N1 ${ }_{\text {pan }}$ ) stem (cH6/1) or A/Anhui/1-YK/2013 (A/H7/N2) in the context of A/ 
Perth/16/2009 (A/H3N2) stem, as previously described $(78,79)$. Correct stem conformation was validated by ELISA using CR6261 or CR8020 stem-directed monoclonal antibodies. WT and chimeric rHA proteins were expressed in EXPI293 cells and purified via a C-terminal histidine tag using HisTrap excel nickel-affinity chromatography columns (GE Healthcare Life Sciences) as previously described (79). Purified rHA proteins were dialyzed against PBS, and total protein concentration was adjusted to $\sim 1 \mathrm{mg} / \mathrm{mL}$ after BCA estimation.

ELISA. Immulon 4HBX plates (Thermo Fisher Scientific) were coated with $50 \mathrm{ng} /$ well of rHA in carbonate buffer ( $\mathrm{pH} 9.4$ ) with $250 \mathrm{ng} / \mathrm{mL}$ BSA for $\sim 16$ hours at $4^{\circ} \mathrm{C}$ in humidified chambers. Plates were blocked with blocking buffer ( $2 \%$ BSA, $1 \%$ gelatin in PBS $/ 0.05 \%$ Tween 20 ) at $37^{\circ} \mathrm{C}$ for 2 hours. Serum samples collected from subjects prior to and 21-28 days following vaccination were initially diluted 1:500 and then further serially diluted 1:2 in blocking buffer to generate 7-point binding curves. Serially diluted serum samples were added to the assay plate in duplicate and incubated $\sim 16$ hours overnight at $4^{\circ} \mathrm{C}$ in humidified chambers. Plates were washed 4 times with PBS and HA-specific IgG detected using HRP-conjugated goat anti-human IgG (Southern Biotech; catalog 2040-05) at a 1:4000 dilution and incubated for 2 hours at $37^{\circ} \mathrm{C}$. Plates were then washed 7 times with PBS prior to development with $100 \mu \mathrm{L}$ of $0.1 \%$ 2,2'-azino-bis(3-ethylbenzothiazoline-6-sulphonic acid) (ABTS) solution with $0.05 \% \mathrm{H}_{2} \mathrm{O}_{2}$ for 20 minutes at $37^{\circ} \mathrm{C}$. The reaction was terminated with $1 \%(\mathrm{w} / \mathrm{v})$ sodium dodecyl sulfate (SDS). Colorimetric absorbance at $414 \mathrm{~nm}$ was measured using a PowerWaveXS plate reader. HA-specific IgG equivalent concentration was calculated based on an 8-point standard curve generated using a human IgG reference protein (Athens Research and Technology). Cumulative HA binding was calculated by adding the IgG-equivalent of the 4 vaccine components $\left(\mathrm{H} 1+\mathrm{H} 3+\mathrm{B}_{\text {vic }}+\mathrm{B}_{\text {yam }}\right)$.

Hemagglutination-inhibition (HAI) assay. The hemagglutination inhibition assay was used to measure functional antibodies capable of inhibiting virus agglutination of turkey erythrocytes. The protocols were adapted from the WHO laboratory influenza surveillance manual (43). To characterize contemporary H3N2 strains that have preferential binding to $\alpha(2,6)$-linked sialic acid receptors, the protocols were adapted from the WHO laboratory influenza surveillance manual (43). An equal volume of each H3N2 virus, adjusted to approximately 8 hemagglutination units (HAU)/50 $\mu \mathrm{L}$ in the presence of $20 \mathrm{nM}$ Oseltamivir (AOBIOUS), was added to each well. The plates were covered and incubated at room temperature (RT) for 30 minutes, and then $0.75 \%$ guinea pig erythrocytes (Lampire Biologicals) in PBS were added.

To inactivate nonspecific inhibitors, sera were treated with receptor-destroying enzyme (RDE) (Denka Seiken Co.) prior to being tested. Briefly, 3 parts of RDE were added to 1 part of sera and incubated overnight at $37^{\circ} \mathrm{C}$. RDE was inactivated by incubation at $56^{\circ} \mathrm{C}$ for $30-45$ minutes and then cooled to RT before diluting with $1 \times \mathrm{PBS}$ or $0.85 \% \mathrm{NaCl}$ to a final sera concentration of $1: 10$. RDE-treated sera was serially diluted in PBS 2-fold across v-bottom microtiter plates (Greiner Bio-One). An equal volume of each influenza virus $(25 \mu \mathrm{L})$, adjusted to a concentration of $\sim 8 \mathrm{HAU} / 50 \mu \mathrm{L}$, was added to each well. The plates were covered and incubated at RT for 20 minutes, and then erythrocytes (Lampire Biologicals) in PBS were added. $\mathrm{RBC}$ s were stored at $4^{\circ} \mathrm{C}$ and used within 72 hours of preparation. The plates were mixed by agitation and covered, and the RBCs settled for 30 minutes at RT. The HAI titer was determined by the reciprocal dilution of the last well that contained nonagglutinated RBCs. Positive and negative serum controls were included for each plate. Seroprotection was defined as HAI titer $\geq 1: 40$ and seroconversion was defined as a 4-fold increase in titer compared with baseline, resulting in a titer of $\geq 1: 40$, as per the WHO and European Committee for Medicinal Products to evaluate influenza vaccines (43). People were considered seronegative with a titer less than 1:40.

Flow cytometry. Human PBMC $\left(\sim 5 \times 10^{6}\right.$ live cells $)$ were stained on ice for 30 minutes with $100 \mu \mathrm{L}$ of staining buffer (PBS/2\% FBS). Human PBMC were first treated with Fc receptor blocking solution (BioLegend) and then stained for 30 minutes on ice using titrated quantities of fluorescently conjugated monoclonal antibodies (Supplemental Table 1). After completion of surface labeling, human PBMC were washed extensively with staining buffer prior to fixation with $1.6 \%$ paraformaldehyde in staining buffer for 15 minutes at RT. Following fixation, cells were pelleted by centrifugation at $400 \mathrm{~g}$ for 5 minutes, resuspended in staining buffer, and maintained at $4^{\circ} \mathrm{C}$ protected from light until acquisition. Data acquisition was performed using the BD FACSARIA Fusion and analysis performed using FlowJo (FlowJo LLC). Compensation values were established prior to acquisition using appropriate single stain controls. Plasmablasts were defined as CD3/CD14- $\mathrm{CD} 19^{+}, \mathrm{CD} 27^{+}, \mathrm{CD} 38^{++}$cells, as previously described $(80,81)$.

In vitro differentiation of $B$ cells. PBMC were cultured $\left(2 \times 10^{6}\right.$ viable cells $\left./ \mathrm{mL}\right)$ in complete media containing RPMI 1640 medium (MilliporeSigma) with 10\% FBS (Atlanta Biologicals), $23.8 \mathrm{mM}$ sodium bicarbonate (Thermo Fisher Scientific), $7.5 \mathrm{mM}$ HEPES (Amresco), $170 \mu \mathrm{M}$ Penicillin G (Tokyo Chemical 
Industry), $137 \mu \mathrm{M}$ Streptomycin (MilliporeSigma), $50 \mu \mathrm{M}$ 2-mercaptoethanol (MilliporeSigma), $1 \mathrm{mM}$ sodium pyruvate (Thermo Fisher Scientific), essential amino acid solution (Thermo Fisher Scientific), nonessential amino acid solution (Thermo Fisher Scientific), $500 \mathrm{ng} / \mathrm{mL}$ R848 (Invivogen), and $5 \mathrm{ng} / \mathrm{mL} \mathrm{rIL}-2$ (R\&D Systems) for $7-9$ days at $37^{\circ} \mathrm{C}$ in $5 \% \mathrm{CO}_{2}(82,83)$. Conditioned medium supernatants were harvested and evaluated for total and rHA-specific IgG abundance by ELISA starting at a 1:5 dilution. Frequency of B cells among total viable PBMC was assessed by CD19 surface labeling and flow cytometry analysis.

ELISpot. MultiScreen ${ }_{\mathrm{HTS}}$ HA filter ELISpot plates (MilliporeSigma) were coated overnight at $4^{\circ} \mathrm{C}$ with $50 \mu \mathrm{L} /$ well of carbonate buffer ( $\mathrm{pH}$ 9.4) containing $1 \mu \mathrm{g} / \mathrm{mL}$ of $\mathrm{rHA}$ and $5 \mu \mathrm{g} / \mathrm{mL}$ BSA in a humidified chamber. Alternatively, ELISpot plates were coated $\sim 16$ hours overnight with $50 \mu \mathrm{L} /$ well of carbonate buffer ( $\mathrm{pH}$ 9.4) containing $5 \mu \mathrm{g} / \mathrm{mL}$ goat anti-human Ig (Southern Biotech; catalog 2070-01) and $5 \mu \mathrm{g}$ goat anti-human Ig (Southern Biotech; catalog 2060-01). Additional plates were coated with carbonate buffer ( $\mathrm{pH}$ 9.4) containing $5 \mu \mathrm{g} / \mathrm{mL}$ BSA alone for background subtraction. Serially diluted human PBMC were incubated in ELISpot plates overnight ( $\sim 16$ hours) prior to washing 3 times with PBS $+0.1 \%$ Triton X-100 (MilliporeSigma). After an additional 3 washes with PBS, biotinylated goat anti-human IgG (Southern Biotech; catalog 2040-05) (diluted 1:5,000) was added (50 $\mu \mathrm{L} /$ well), and plates were incubated at $37^{\circ} \mathrm{C}$ for 2 hours. ELISpot plates were then washed 4 times with PBS prior to addition of alkaline phosphatase conjugated streptavidin $(1: 10,000)$ and incubated at $37^{\circ} \mathrm{C}$ for 1 hour. Plates were then washed 5 times with PBS and 3 times with distilled water prior to addition of 5-bromo-4-chloro-3-indolyl phosphate (BCIP)/nitro blue tetrazolium (NBT) 1-step solution (Thermo Fisher Scientific) $\left(50 \mu \mathrm{L} /\right.$ well) and incubation at $37^{\circ} \mathrm{C}$ for 15-20 minutes. Development of the BCIP/NBT substrate was terminated by decanting the plates and washing 3 times with distilled water. Plates were then air dried at RT prior to image acquisition using the S6 macro ELISpot reader and automated counting using ImmunoSpot (v5.1) software (Cellular Technology Limited). Number of ASC was normalized to $1 \times 10^{5}$ PBMCs. Percentage of HA-specific PB response to each vaccine strain was calculated from the cumulative number of ASC against the 4 vaccine components $\left(\mathrm{H} 1+\mathrm{H} 3+\mathrm{B}_{\text {vic }}+\mathrm{B}_{\text {yam }}\right)$.

Supplemental materials. HAI immunodominance, serological assay correlations, flow cytometry gating strategies and extra $\mathrm{B}_{\text {mem }}$-derived antibody binding data can be found in Supplemental Figure 1, the Supplemental Tables, and Supplemental Figure 3, respectively.

Statistics. Subjects were grouped by age as previously described (77) or serological changes in HAI

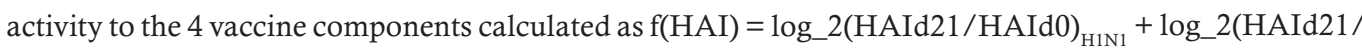
HAId0 $)_{\mathrm{H} 3 \mathrm{~N} 2}+\log \__{2}(\mathrm{HAId} 21 / \mathrm{HAId} 0)_{\text {Bvic }}+\log \__{2}(\mathrm{HAId} 21 / \mathrm{HAId} 0)_{\text {Byam }}$. Subjects with $\mathrm{f}(\mathrm{HAI})<4$ were defined as nonresponders, subjects with $4<\mathrm{f}(\mathrm{HAI})<8$ were defined as low responders and subjects $\mathrm{f}(\mathrm{HAI})>8$ were defined as high responders.

The response to each individual vaccine component was categorized as per the WHO and European Committee for Medicinal Products to evaluate influenza vaccines (43). Minimal seroprotection was defined as HAI titer of 1:40 to 1:80 (low responders) and high seroprotection as HAI titer > 1:80 (High responders). Minimal seroconversion was defined as a 4- to 8-fold increase in HAI titer compared with baseline, resulting in a titer of $\geq 1: 40$ and high serconversion defined as $>8$-fold rise in HAI titer. Subjects were considered seronegative with a titer less than 1:40.

Statistical significance between groups was calculated using 1-way ANOVA Friedman test and Dunn's multiple comparisons. Values were considered significant for $P<0.05$. Unless otherwise stated, data is presented from at least 3 independent experiments.

Percentage of HA binding to each vaccine strain was calculated from the cumulative IgG binding to the 4 vaccine components for each subject individually $\left(\mathrm{H} 1+\mathrm{H} 3+\mathrm{B}_{\text {vic }}+\mathrm{B}_{\text {yam }}\right)$. For percentage of HAI activity, serum titers were transformed to a $\log _{2}$ scale prior to calculation, to avoid skewness.

Correlation analyses between HAI titer and HA-binging $\operatorname{IgG}(\mu \mathrm{g} / \mathrm{mL})$ in the serum or from $\mathrm{B}_{\text {mem }}$-differenteiated cultures was performed by nonparametric Spearman correlation.

Significant immunodominance in a group was calculated by one-sample Wilcoxon signed rank test (where $\% \mathrm{HA} \neq 25)$ and 1-way ANOVA Friedman test and Dunn's multiple comparisons $\left(\mathrm{H} 1 \neq \mathrm{H} 3 \neq \mathrm{B}_{\mathrm{vic}} \neq \mathrm{B}_{\text {yam }}\right)$. Statistical significance $(P<0.05)$ must be reached in both tests, and the highest $P$ value is represented. Differences between pre- and postvaccination data were calculated by 1-way ANOVA multiple comparisons.

Significant immunodominance for each donor was assessed by 2 independent multiple $t$-test one per donor. Replicate readings $(n \geq 6)$ of HA-specific IgG and percentage of HA binding were tested for significant differences between vaccine components $\left(\mathrm{H} 1 \neq \mathrm{H} 3 \neq \mathrm{B}_{\text {vic }} \neq \mathrm{B}_{\text {yam }}\right.$ and $\left.\% \mathrm{H} 1 \neq \% \mathrm{H} 3 \neq \% \mathrm{~B}_{\text {vic }} \neq \% \mathrm{~B}_{\text {yam }}\right)$. 
Statistical significance $(P<0.05)$ must be reached in both tests. Subjects with readings below limit of detection were excluded from analysis.

All statistical analyses were performed using GraphPad Prism V.8.01 software.

Study approval. The study procedures, informed consent, and data collection documents were reviewed and approved by the IRB of the University of Georgia. The funding source had no role in sample collection, nor decision to submit the manuscript for publication. Subjects were recruited at the UGA Clinical Trials Research Unit (CTRU) in Athens, Georgia, USA, and enrolled with written, informed consent. Exclusion criteria included documented contraindications to Guillain-Barré syndrome, dementia or Alzheimer's disease, allergies to eggs or egg products, estimated life expectancy $<2$ years, medical treatment causing or diagnosis of an immunocompromising condition, or concurrent participation in another influenza vaccine research study. Influenza virus did not circulate widely in the community during the time periods that the subjects participated, and as such, participants were not monitored for influenza virus infection during that time period. However, study participants were asked during each visit if they had experienced flu-like symptoms, and those who did were excluded from the study.

\section{Author contributions}

RBA conceptualized and wrote the manuscript, designed and performed experiments and analyzed data; GAK conceptualized and edited the manuscript, and designed and performed experiments; EFC performed experiments and curated data; GAS designed and performed experiments and edited the manuscript; TMR acquired funding for, helped to conceptualize, and edited the manuscript.

\section{Acknowledgments}

The authors would like to thank Spencer R. Pierce and Jeffrey W. Ecker for protein expression and purification, as well as Ivette A. Nuñez, Thomas M. Rowe, James D. Allen, Bradford C. Lefoley, Simon O. Owino, Krissy K. Moehling, Patricia Nowalk, Michael Susick, Kensington Diagle, and Kristen Sweeney for technical assistance. We would also like to thank Anne G. Bebin-Blackwell and Harry Kleanthous for helpful discussions and comments. We also thank Jonathan Murrow, Brad Phillips, Kim Schmitz, and the members of the UGA Clinical Trials Evaluation Unit. We also give a special thanks and appreciation to the volunteer subjects who participated in the study. The following reagents were obtained through the NIH Biodefense and Emerging Infections Research Resources Repository, NIAID, NIH: MDCK; Kidney (canine), Working Cell Bank, NR-2628. This work was partially supported by the National Center for Advancing Translational Sciences of the NIH under award number UL1TR002378, and Emory-UGA Center of Excellence of Influenza research and Surveillance (Emory-UGA CEIRS) contract grant (HHSN272201400004C). The content is solely the responsibility of the authors and does not necessarily represent the official views of the NIH.

Address correspondence to: Ted M. Ross, Center for Vaccines and Immunology, University of Georgia, 501 D.W. Brooks Drive, CVI Room 1504, Athens, Georgia 30602, USA. Phone: 706.542.9708; Email: tedross@uga.edu.

GAK's present address is: Cellular Technology Limited (CTL), Shaker Heights, Ohio, USA.

1. [No authors listed]. CDC/NCIRD C for DC P. Estimated Influenza Illnesses, Medical visits, Hospitalizations, and Deaths in the United States - 2017-2018 influenza season. Centers for Disease Control and Prevention. https://www.cdc.gov/flu/ about/burden/2017-2018.htm. Accessed November 27, 2019.

2. Cai S, Temkin-Greener H. Influenza vaccination and its impact on hospitalization events in nursing homes. J Am Med Dir Assoc. 2011;12(7):493-498.

3. Falsey AR, McElhaney JE. Influenza burden in frail elderly. Lancet Respir Med. 2018;6(1):e2.

4. Simonsen L, Reichert TA, Viboud C, Blackwelder WC, Taylor RJ, Miller MA. Impact of influenza vaccination on seasonal mortality in the US elderly population. Arch Intern Med. 2005;165(3):265-272.

5. Iuliano $\mathrm{AD}$, et al. Estimates of global seasonal influenza-associated respiratory mortality: a modelling study. Lancet. 2018;391(10127):1285-1300.

6. Blomberg BB, Frasca D. Quantity, not quality, of antibody response decreased in the elderly. J Clin Invest. 2011;121(8):2981-2983

7. Souty C, et al. Early estimates of 2014/15 seasonal influenza vaccine effectiveness in preventing influenza-like illness in general practice using the screening method in France. Hum Vaccin Immunother. 2015;11(7):1621-1625.

8. Goodwin K, Viboud C, Simonsen L. Antibody response to influenza vaccination in the elderly: a quantitative review. Vaccine. 2006;24(8):1159-1169

9. Osterholm MT, Kelley NS, Sommer A, Belongia EA. Efficacy and effectiveness of influenza vaccines: a systematic review and meta-analysis. Lancet Infect Dis. 2012;12(1):36-44. 
10. Caton AJ, Brownlee GG, Yewdell JW, Gerhard W. The antigenic structure of the influenza virus A/PR/8/34 hemagglutinin (H1 subtype). Cell. 1982;31(2 Pt 1):417-427.

11. Krystal M, Elliott RM, Benz EW, Young JF, Palese P. Evolution of influenza A and B viruses: conservation of structural features in the hemagglutinin genes. Proc Natl Acad Sci USA. 1982;79(15):4800-4804.

12. Ni F, Kondrashkina E, Wang Q. Structural basis for the divergent evolution of influenza B virus hemagglutinin. Virology. 2013;446(1-2):112-122.

13. Nuñez IA, et al. Impact of age and pre-existing influenza immune responses in humans receiving split inactivated influenza vaccine on the induction of the breadth of antibodies to influenza A strains. PLoS One. 2017;12(11):e0185666.

14. Park JK, et al. Evaluation of Preexisting Anti-Hemagglutinin Stalk Antibody as a Correlate of Protection in a Healthy Volunteer Challenge with Influenza A/H1N1pdm Virus. MBio. 2018;9(1):e02284-17.

15. Jacobsen H, et al. Influenza Virus Hemagglutinin Stalk-Specific Antibodies in Human Serum are a Surrogate Marker for In Vivo Protection in a Serum Transfer Mouse Challenge Model. MBio. 2017;8(5):e0146317.

16. Jegaskanda S, et al. Generation and Protective Ability of Influenza Virus-Specific Antibody-Dependent Cellular Cytotoxicity in Humans Elicited by Vaccination, Natural Infection, and Experimental Challenge. J Infect Dis. 2016;214(6):945-952.

17. Tan HX, et al. Subdominance and poor intrinsic immunogenicity limit humoral immunity targeting influenza HA stem. $J$ Clin Invest. 2019;129(2):850-862.

18. GlaxoSmithKline. A Study to Evaluate the Reactogenicity, Safety and Immunogenicity of GlaxoSmithKline (GSK) Biologicals' Investigational Supra-seasonal Universal Influenza Vaccines - Inactivated (SUIVs) (GSK3816302A) in Healthy Adults Aged 18 to 39 Years. National Institutes of Health. https://clinicaltrials.gov/ct2/show/NCT03275389. Published September 7, 2017. Updated September 23, 2019. Accessed November 27, 2019.

19. Yassine HM, et al. Hemagglutinin-stem nanoparticles generate heterosubtypic influenza protection. Nat Med. 2015;21(9):1065-1070

20. Impagliazzo A, et al. A stable trimeric influenza hemagglutinin stem as a broadly protective immunogen. Science. 2015;349(6254):1301-1306.

21. Nachbagauer R, et al. A chimeric haemagglutinin-based influenza split virion vaccine adjuvanted with AS03 induces protective stalk-reactive antibodies in mice. NPJ Vaccines. 2016;1:16015.

22. Hannoun C. The evolving history of influenza viruses and influenza vaccines. Expert Rev Vaccines. 2013;12(9):1085-1094.

23. Maynard JE, Dull HB, Hanson ML, Feltz ET, Berger R, Hammes L. Evaluation of monovalent and polyvalent influenza vaccines during an epidemic of type A2 and B influenza. Am J Epidemiol. 1968;87(1):148-157.

24. Bashe WJ, Stegmiller H, Leonida D, Greenwald P. FAILURE OF POLYVALENT VACCINE TO PROVIDE CLINICAL PROTECTION AGAINST ASIAN INFLUENZA. N Engl J Med. 1964;270:870-874.

25. Barr IG, Jelley LL. The coming era of quadrivalent human influenza vaccines: who will benefit? Drugs. 2012;72(17):2177-2185.

26. Rota PA, Wallis TR, Harmon MW, Rota JS, Kendal AP, Nerome K. Cocirculation of two distinct evolutionary lineages of influenza type B virus since 1983. Virology. 1990;175(1):59-68.

27. Schlingmann B, Castiglia KR, Stobart CC, Moore ML. Polyvalent vaccines: High-maintenance heroes. PLoS Pathog. 2018;14(4):e1006904.

28. Guess HA. Combination vaccines: issues in evaluation of effectiveness and safety. Epidemiol Rev. 1999;21(1):89-95.

29. Smith J, Lipsitch M, Almond JW. Vaccine production, distribution, access, and uptake. Lancet. 2011;378(9789):428-438

30. Robbins JB, et al. Considerations for formulating the second-generation pneumococcal capsular polysaccharide vaccine with emphasis on the cross-reactive types within groups. J Infect Dis. 1983;148(6):1136-1159.

31. Zost SJ, Wu NC, Hensley SE, Wilson IA. Immunodominance and Antigenic Variation of Influenza Virus Hemagglutinin: Implications for Design of Universal Vaccine Immunogens. J Infect Dis. 2019;219(Supplement_1):S38-S45.

32. Lee H, Shim EH, You S. Immunodominance hierarchy of influenza subtype-specific neutralizing antibody response as a hurdle to effectiveness of polyvalent vaccine. Hum Vaccin Immunother. 2018;14(10):2537-2539.

33. Huang KYA et al. Focused antibody response to influenza linked to antigenic drift. J Clin invest. 2015;125(7):2631-2645.

34. Kreijtz JH, Fouchier RA, Rimmelzwaan GF. Immune responses to influenza virus infection. Virus Res. 2011;162(1-2):19-30.

35. Vatti A, Monsalve DM, Pacheco Y, Chang C, Anaya JM, Gershwin ME. Original antigenic sin: A comprehensive review. J Autoimmun. 2017;83:12-21.

36. Francis T Jr. On the Doctrine of Original Antigenic Sin. Proc Am Philos Soc. 1960;104(6):572-578.

37. Zhang A, Stacey HD, Mullarkey CE, Miller MS. Original Antigenic Sin: How First Exposure Shapes Lifelong Anti-Influenza Virus Immune Responses. J Immunol. 2019;202(2):335-340.

38. Monto AS, Malosh RE, Petrie JG, Martin ET. The Doctrine of Original Antigenic Sin: Separating Good From Evil. J Infect Dis. 2017;215(12):1782-1788.

39. Nachbagauer R, et al. Defining the antibody cross-reactome directed against the influenza virus surface glycoproteins. Nat Immunol. 2017;18(4):464-473.

40. Cobey S, Hensley SE. Immune history and influenza virus susceptibility. Curr Opin Virol. 2017;22:105-111.

41. Sasaki S et al. Limited efficacy of inactivated influenza vaccine in elderly individuals is associated with decreased production of vaccine-specific antibodies. J Clin Invest. 2011;121(8):3109-3119.

42. Abreu R, Ross TM. Influenza - A new pathogen every year. Curr Opin Syst Biol. 2018;12:12-21.

43. WHO Global Influenza Surveillance Network. Manual for the laboratory diagnosis and virological surveillance of influenza. World Health Organization. https://www.who.int/influenza/gisrs_laboratory/manual_diagnosis_surveillance_influenza/en/. Accessed November 27, 2019.

44. Izurieta HS, et al. Comparative effectiveness of high-dose versus standard-dose influenza vaccines in US residents aged 65 years and older from 2012 to 2013 using Medicare data: a retrospective cohort analysis. Lancet Infect Dis. 2015;15(3):293-300.

45. Global Influenza Market: Size, Trends and Forecast (2018-2022) with GlaxoSmithKline, AstraZeneca, Thermo Fisher Scientific, and Abbott Laboratories Dominating [new release]. Dublin: Business Wire; December 3, 2018. https://www.businesswire.com/ news/home/20181203005430/en/Global-Influenza-Market-Size-Trends-Forecast-2018-2022. Accessed November 27, 2019.

46. [No authors listed]. Age Group Distribution of Influenza Positive Specimens Reported by Public Health Laboratories, National Summary, 2019-20 Influenza Season through the Week Ending November 16, 2019. Center for Disease Control and Prevention. 
https://gis.cdc.gov/grasp/fluview/flu_by_age_virus.html. Accessed November 27, 2019.

47. Blanton L, et al. Update: Influenza Activity - United States, October 2, 2016-February 4, 2017. MMWR Morb Mortal Wkly Rep 2017;66(6):159-166

48. Thompson WW, et al. Mortality associated with influenza and respiratory syncytial virus in the United States. JAMA. 2003;289(2):179-186

49. DiazGranados CA, et al. Efficacy of high-dose versus standard-dose influenza vaccine in older adults. $N$ Engl J Med. 2014;371(7):635-645.

50. Ng S, et al. The effect of age and recent influenza vaccination history on the immunogenicity and efficacy of 2009-10 seasonal trivalent inactivated influenza vaccination in children. PLoS One. 2013;8(3):e59077.

51. [No authors listed]. Seasonal Influenza Vaccine Effectiveness, 2016-2017. Centers for Disease Prevention and Control. https:// www.cdc.gov/flu/vaccines-work/2016-2017.html. Accessed November 27, 2019.

52. Monto AS, Maassab HF. Ether treatment of type B influenza virus antigen for the hemagglutination inhibition test. J Clin Microbiol. 1981;13(1):54-57.

53. Kendal AP, Cate TR. Increased sensitivity and reduced specificity of hemagglutination inhibition tests with ether-treated influenza B/Singapore/222/79. J Clin Microbiol. 1983;18(4):930-934.

54. Bedford T, et al. Integrating influenza antigenic dynamics with molecular evolution. Elife. 2014;3:e01914.

55. Suptawiwat O, Ninpan K, Boonarkart C, Ruangrung K, Auewarakul P. Evolutionary dynamic of antigenic residues on influenza B hemagglutinin. Virology. 2017;502:84-96.

56. Berton MT, Webster RG. The antigenic structure of the influenza B virus hemagglutinin: operational and topological mapping with monoclonal antibodies. Virology. 1985;143(2):583-594.

57. Hovanec DL, Air GM. Antigenic structure of the hemagglutinin of influenza virus B/Hong Kong/8/73 as determined from gene sequence analysis of variants selected with monoclonal antibodies. Virology. 1984;139(2):384-392.

58. Wiley DC, Wilson IA, Skehel JJ. Structural identification of the antibody-binding sites of Hong Kong influenza haemagglutinin and their involvement in antigenic variation. Nature. 1981;289(5796):373-378.

59. Gerhard W, Yewdell J, Frankel ME, Webster R. Antigenic structure of influenza virus haemagglutinin defined by hybridoma antibodies. Nature. 1981;290(5808):713-717.

60. Berton MT, Naeve CW, Webster RG. Antigenic structure of the influenza B virus hemagglutinin: nucleotide sequence analysis of antigenic variants selected with monoclonal antibodies. J Virol. 1984;52(3):919-927.

61. Krystal M, Young JF, Palese P, Wilson IA, Skehel JJ, Wiley DC. Sequential mutations in hemagglutinins of influenza B virus isolates: definition of antigenic domains. Proc Natl Acad Sci USA. 1983;80(14):4527-4531.

62. Potter CW, Oxford JS. Determinants of immunity to influenza infection in man. Br Med Bull. 1979;35(1):69-75.

63. Pillet S, et al. Cellular immune response in the presence of protective antibody levels correlates with protection against 1918 influenza in ferrets. Vaccine. 2011;29(39):6793-6801.

64. Gould VMW, Francis JN, Anderson KJ, Georges B, Cope AV, Tregoning JS. Nasal IgA Provides Protection against Human Influenza Challenge in Volunteers with Low Serum Influenza Antibody Titre. Front Microbiol. 2017;8:900.

65. Sridhar S, et al. Cellular immune correlates of protection against symptomatic pandemic influenza. Nat Med. 2013;19(10):13051312 .

66. Sasaki S, et al. Comparison of the influenza virus-specific effector and memory B-cell responses to immunization of children and adults with live attenuated or inactivated influenza virus vaccines. J Virol. 2007;81(1):215-228.

67. Bongen E, Vallania F, Utz PJ, Khatri P. KLRD1-expressing natural killer cells predict influenza susceptibility. Genome Med. 2018;10(1):45

68. Li GM, et al. Pandemic H1N1 influenza vaccine induces a recall response in humans that favors broadly cross-reactive memory B cells. Proc Natl Acad Sci USA. 2012;109(23):9047-9052.

69. Kosikova M, Li L, Radvak P, Ye Z, Wan XF, Xie H. Imprinting of Repeated Influenza A/H3 Exposures on Antibody Quantity and Antibody Quality: Implications for Seasonal Vaccine Strain Selection and Vaccine Performance. Clin Infect Dis. 2018;67(10):1523-1532.

70. Choi YS, et al. Reduced antibody responses to the pandemic (H1N1) 2009 vaccine after recent seasonal influenza vaccination. Clin Vaccine Immunol. 2011;18(9):1519-1523.

71. Andrews SF, et al. Immune history profoundly affects broadly protective B cell responses to influenza. Sci Transl Med. 2015;7(316):316ra192.

72. Cheng X, et al. Evaluation of the humoral and cellular immune responses elicited by the live attenuated and inactivated influenza vaccines and their roles in heterologous protection in ferrets. J Infect Dis. 2013;208(4):594-602.

73. Lang S, Xie J, Zhu X, Wu NC, Lerner RA, Wilson IA. Antibody 27F3 Broadly Targets Influenza A Group 1 and 2 Hemagglutinins through a Further Variation in $\mathrm{V}_{\mathrm{H}} 1-69$ Antibody Orientation on the HA Stem. Cell Rep. 2017;20(12):2935-2943.

74. Ellebedy $\mathrm{AH}$, et al. Induction of broadly cross-reactive antibody responses to the influenza HA stem region following H5N1 vaccination in humans. Proc Natl Acad Sci USA. 2014;111(36):13133-13138.

75. Nachbagauer R, et al. A universal influenza virus vaccine candidate confers protection against pandemic H1N1 infection in preclinical ferret studies. NPJ Vaccines. 2017;2:26.

76. Zost SJ, et al. Contemporary H3N2 influenza viruses have a glycosylation site that alters binding of antibodies elicited by egg-adapted vaccine strains. Proc Natl Acad Sci USA. 2017;114(47):12578-12583.

77. Carlock MA, et al. Impact of age and pre-existing immunity on the induction of human antibody responses against influenza $B$ viruses. Hum Vaccin Immunother. 2019;15(9):2030-2043

78. Kirchenbaum GA, Allen JD, Layman TS, Sautto GA, Ross TM. Infection of Ferrets with Influenza Virus Elicits a Light Chain-Biased Antibody Response against Hemagglutinin. J Immunol. 2017;199(11):3798-3807.

79. Sautto GA, Kirchenbaum GA, Ecker JW, Bebin-Blackwell AG, Pierce SR, Ross TM. Elicitation of Broadly Protective Antibodies following Infection with Influenza Viruses Expressing H1N1 Computationally Optimized Broadly Reactive Hemagglutinin Antigens. Immunohorizons. 2018;2(7):226-237.

80. Perez-Andres M, et al. Human peripheral blood B-cell compartments: a crossroad in B-cell traffic. Cytometry B Clin Cytom. 
2010;78 Supp1 1:S47-S60.

81. Sanz I, Wei C, Lee FE, Anolik J. Phenotypic and functional heterogeneity of human memory B cells. Semin Immunol. 2008;20(1):67-82.

82. Faenzi E, et al. One dose of an MF59-adjuvanted pandemic A/H1N1 vaccine recruits pre-existing immune memory and induces the rapid rise of neutralizing antibodies. Vaccine. 2012;30(27):4086-4094.

83. Frasca D, Diaz A, Romero M, Blomberg BB. The generation of memory B cells is maintained, but the antibody response is not, in the elderly after repeated influenza immunizations. Vaccine. 2016;34(25):2834-2840. 